TRANSACTIONS OF THE

AMERICAN MATHEMATICAL SOCIETY

Volume 361, Number 4, April 2009, Pages 2021-2046

S 0002-9947(08)04778-8

Article electronically published on November 25, 2008

\title{
WORMHOLES IN ACH EINSTEIN MANIFOLDS
}

\author{
OLIVIER BIQUARD AND YANN ROLLIN
}

\begin{abstract}
We give a new construction of Einstein manifolds which are asymptotically complex hyperbolic, inspired by the work of Mazzeo-Pacard in the real hyperbolic case. The idea is to develop a gluing theorem for 1-handle surgery at infinity, which generalizes the Klein construction for the complex hyperbolic metric.
\end{abstract}

\section{INTRODUCTION}

In this paper, we present a new construction of asymptotically complex hyperbolic Einstein metrics (we shall use the acronym ACH from now on) by gluing wormholes on their conformal infinity. Our results extend the work of Mazzeo and Pacard 12 in the context of asymptotically real hyperbolic Einstein metrics. Using our gluing theory, we can produce many new examples of $\mathrm{ACH}$ Einstein metrics. An interesting feature of the "complex hyperbolic" theory is that it also enables us to construct Kähler-Einstein metrics as well.

1.1. Statement of results. First, let us recall the concept of an ACH metric: let $\bar{X}$ be a compact manifold of even dimension $m=2 n$ with boundary $Y$. We will denote by $X$ the interior of $\bar{X}$, and choose a defining function $u$ of $Y$ that is a function on $\bar{X}$, positive on $X$ and vanishing to first order on $Y=\partial X$.

The notion of an ACH metric on $X$ is related to the data of a strictly pseudoconvex CR structure on $Y$, that is an almost complex structure $J$ on a contact distribution of $Y$, such that $\gamma(\cdot, \cdot)=d \eta(\cdot, J \cdot)$ is a positive Hermitian metric on the contact distribution (here we have chosen a contact form $\eta$ ).

Identify a collar neighborhood of $Y$ in $\bar{X}$ with $[0, T) \times Y$, with coordinate $u$ on the first factor. A Riemannian metric $g$ is defined to be an $\mathrm{ACH}$ metric on $X$ if there exists a CR structure $J$ on $Y$, such that near $Y$,

$$
g \sim \frac{d u^{2}+\eta^{2}}{u^{2}}+\frac{\gamma}{u}
$$

in a sense which will be made precise in Section 3.2 (observe that for $J$ being the standard invariant CR structure of the Heisenberg group, the RHS of (1.1) is exactly the complex hyperbolic metric). The manifold $(Y, J)$ is called the conformal infinity of $(X, g)$.

We will also consider more general $\mathrm{ACH}$ metrics by allowing $(\eta, J)$ to be defined only up to sign. If $n$ is even, notice that $\operatorname{vol}^{Y}:=\eta \wedge(d \eta)^{n-1}$ is well defined although

Received by the editors May 9, 2007.

2000 Mathematics Subject Classification. Primary 32Q20, 53C25.

The second author was partly supported by a University Research Fellowship of the Royal Society and NSF grant \#DMS-0305130.

(c)2008 American Mathematical Society Reverts to public domain 28 years from publication 
$\eta$ is defined up to sign. It follows that the contact structure induces a standard orientation on $Y$ given by $\operatorname{vol}^{Y}$ if $\operatorname{dim}_{\mathbb{R}} Y=3 \bmod 4$. If $n$ is odd (i.e. $\operatorname{dim}_{\mathbb{R}} Y=1$ mod 4), then neither the contact distribution nor $Y$ need to be orientable. However, an orientation for $Y$ determines an orientation for $\xi$ and vice versa.

We can now state our main theorem:

Theorem A. Let $\bar{X}$ be a compact m-dimensional manifold with boundary and $m=2 n$, such that its interior $X$ is endowed with an unobstructed ACH Einstein metric. Let $\bar{X}_{k}:=\bar{X} \cup k\left(B^{1} \times B^{m-1}\right)$ be the manifold obtained by gluing $k$ copies of a 1-handle on the boundary of $\bar{X}$. If 4 divides $m$, we require moreover that the handle additions are compatible with the contact orientation of the boundary.

Then the interior $X_{k}$ of $\bar{X}_{k}$ carries an unobstructed ACH Einstein metric.

Remark 1.1.1. The existence of an ACH metric in the conclusion of Theorem A implies that there exists a strictly pseudoconvex CR structure on the boundary of $\bar{X}_{k}$, i.e. the conformal infinity of the metric. Thus we recover a result already known for more general surgeries (cf. 9], 14]).

At the moment, Theorem $\mathrm{A}$ is stated in a rather imprecise way. The metrics on $X_{k}$ are in fact obtained by a gluing theorem: given $X$ endowed on an $\mathrm{ACH}$ Einstein metric, we construct a sequence of approximate Einstein metrics on $X_{k}$ (see Section 3).

These approximate Einstein metrics come as a family parametrized by $\mathbb{R}^{2} \times$ $U(n-1)$ (in the case $k=1$ ), and so do the metrics produced in Theorem $\mathrm{A}$. This will be clear from the technical version of this result given in Theorem 4.4.1,

The obstruction hypothesis will be defined later. It is expected to be quite generic and is used to deform the approximate solutions into true Einstein metrics. At this point, all we need to know is that there are three important cases where the obstruction vanishes; see Section 4.5.

Proposition B. Assume that $X$ is endowed with an ACH Einstein metric $g$ and either

- $g$ has negative sectional curvature,

- $X$ is oriented, 4-dimensional and $g$ is self-dual Einstein, or

- $X$ is a complex manifold, the metric $g$ is Kähler-Einstein and the compactly supported cohomology group $H_{c}^{1}(X, T X)$ vanishes;

then there is no obstruction. Moreover, if $X$ is a disjoint union of unobstructed components, it is unobstructed.

At the moment, the only known ACH Einstein metrics of negative sectional curvature are the complex hyperbolic metrics and their deformations constructed in 2. It is very important to know that they are unobstructed. In particular, it implies that the 1-handles identified to $B^{m}$ with its Bergman metric is unobstructed; this property turns out to be essential for the proof of Theorem $\mathrm{A}$.

However, the gluing problem for gluing Kähler-Einstein metrics is automatically unobstructed, once the complex structure is fixed. Thus we obtain the following variation on Theorem $\mathrm{A}$ for the $\mathrm{ACH}$ Kähler-Einstein metric.

Proposition C. Let $\bar{X}$ be a compact complex manifold with boundary and $\operatorname{dim}_{\mathbb{C}} M$ $=n$, such that its interior $X$ is endowed with an ACH Kähler-Einstein metric. Let $\bar{X}_{k}:=\bar{X} \cup k\left(B^{1} \times B^{2 n-1}\right)$ be the manifold obtained by adding $k$ copies of a 1-handle respecting the complex orientation. 
Then the interior $\bar{X}_{k}$ can be endowed with a complex structure and its interior $X_{k}$ carries an ACH Kähler-Einstein metric.

Again the precise technical version of this theorem will be given in Section 4.4 .

1.2. Applications. Existence of ACH (Kähler)-Einstein metrics is known in several cases:

(1) Complex hyperbolic quotients: some (infinite volume) quotients of $\mathbb{C} \mathcal{H}^{n}$ by a group of isometries are $\mathrm{ACH}$, for example disk bundles $\sqrt{T \Sigma}$ over a hyperbolic Riemann surface $\Sigma$ come from a representation of the fundamental group $\Sigma$ into $S U(1,1) \subset S U(n, 1)$. On these $\mathrm{ACH}$ complex hyperbolic metrics one can perform the so called Klein construction (see Section 3.5): for instance, the Klein construction on the Bergman ball corresponds topologically to glue a 1-handle. This is precisely the construction that we generalize in Theorem A.

(2) Kähler-Einstein metrics: a strictly pseudoconvex domain of $\mathbb{C}^{n}$ carries an $\mathrm{ACH}$ Kähler-Einstein metric, the Cheng-Yau metric constructed in [6], whose prototypical example is the Bergman metric on the ball (see also [13]); other examples include a small neighborhood of the zero section of the cotangent bundle of a real analytic manifold.

(3) Self-dual Einstein metrics: Calderbank and Singer consider the minimal resolutions $X$ of the quotient singularity $\mathbb{C}^{2} / \Gamma$ such that $c_{1}(X)<0$, where $\Gamma$ is a finite cyclic subgroup of $U(2)$. In [5] they find an ansatz for an $\mathrm{ACH}$ self-dual Einstein metric defined on a neighborhood of the exceptional fiber in $X$, with conformal infinity the link of the singularity. For example, the unit disk bundle $D(-p)$ of $O(-p) \rightarrow \mathbb{C P}^{1}$ carries an ACH Einstein metric for $p \geq 3$.

These metrics are starting points for the application of Theorem $\mathrm{A}$ to get new $\mathrm{ACH}$ Einstein metrics. It also gives new light on the problem: which manifolds carry strictly pseudoconvex CR structure arising as the conformal infinity of an ACH Einstein metric?

To answer this question, Theorem $\mathrm{A}$ is not useful when applied to an $\mathrm{ACH}$ Einstein metric of negative sectional curvature. At the moment, the only known metrics with this property are the complex hyperbolic examples and their deformations. However we can directly perform the handle addition in this case (see Section 3.5), and Theorem $\mathrm{A}$ is not really needed.

The case of self-dual Einstein metrics is much more enticing. We mentioned earlier the large class of ACH self-dual Einstein metrics constructed by Calderbank and Singer. Since they are unobstructed by Proposition B we can add 1-handles to these spaces and get many new ACH Einstein metrics. Rather than describing the complete list of all possible examples one can get in this way, we just give a very particular case and let the interested reader consult [5] and experiment on his own: the boundary connected sum

$$
\bar{X}_{1}=\overline{D(-p)} \sharp_{b} \overline{D(-q)}
$$

is obtained by adding a 1-handle to the disjoint union $\bar{X}=\overline{D(-p)} \cup \overline{D(-q)}$. For $p, q \geq 3$ it follows from [5], Proposition B and Theorem $\mathrm{A}$ that $X_{1}$ carries an $\mathrm{ACH}$ Einstein metric. Notice that we cannot obtain an Einstein metric by the construction of Cheng-Yau in this case: although $D(-p), D(-q)$ and $X_{1}$ have 
natural complex structures, none of them is a pseudoconvex domain of a Stein manifold since they contain closed curves (the exceptional fibers).

We can also construct examples of the form $\bar{X}_{1}=\overline{D(-p)} \sharp_{b} \bar{Z}$, where $p \geq 3$ and $Z$ is a complex hyperbolic quotient. Then $X_{1}$ carries an ACH Einstein metric. More generally, we can take any disjoint union of complex hyperbolic and $\mathrm{ACH}$ selfdual Einstein manifolds and glue a bunch of 1-handles ad lib. Then, the resulting manifold carries an ACH Einstein metric.

Also note that the absence of obstruction in the Kähler-Einstein case gives a very large class of new ACH Kähler-Einstein manifolds building from the ChengYau metrics on pseudoconvex domains.

There is also a sort of generalization of the Möbius band example: starting from a Cheng-Yau metric on $\bar{X}$, it is possible to build a locally Kähler-Einstein metric on $\bar{X}_{1}$, in the sense that the complex structure $J$ on $\bar{X}_{1}$ is defined only up to sign (see Theorem 4.4.5). These examples admit a double cover which is Kähler-Einstein. This large class of examples of ACH Einstein spaces is fundamentally new.

Finally, the gluing of a 1-handle gives a connected sum for CR structures on the boundary. In the 3-dimensional case, the construction gives some indications on the $\nu$-invariant of 3-dimensional strictly pseudoconvex $\mathrm{CR}$ manifolds introduced in 3]. Recall that this is a kind of $\eta$-invariant for CR manifolds whose gradient when one varies the $\mathrm{CR}$ structure is the Cartan curvature. This means that when the complex structure $J$ varies in a contact distribution, one controls the variation of $\nu$. It is therefore important to understand what is happening when one changes the contact structure. The following proposition is a first step in this direction: it controls what is happening when one performs a simple surgery on the contact structure.

Proposition D. Let $(Y, J)$ be a 3-dimensional strictly pseudoconvex CR manifold. Let $Y^{\sharp}$ be the manifold obtained by applying $k$ successive 1-handle surgeries to $Y$. Then there exists a family of CR structures $\left(J_{\tau}^{\sharp}\right)_{\tau>0}$ on $Y^{\sharp}$, converging to $J$ (away from the surgered locus) when $\tau \rightarrow 0$, such that

$$
\lim _{\tau \rightarrow 0} \nu\left(J_{\tau}^{\sharp}\right)=\nu(J)+k .
$$

In the case where $J$ is spherical, then the CR structure $J_{\tau}$ on $Y_{k}$ can be chosen spherical, and the limit becomes an equality for all $\tau$.

Remark 1.2.1. If $Y$ is connected, $Y_{k}$ is homeomorphic to the connected sum $Y_{k}=$ $Y \sharp k\left(S^{1} \times S^{2}\right)$.

The general path for the proof is close to the one of Mazzeo and Pacard in the real case [12, but in this paper we insist on several interesting new features coming from complex hyperbolic geometry and Kähler geometry. In Section 2 we recall some complex hyperbolic geometry, enabling us to construct approximate solutions on the manifolds obtained by adding a 1-handle. These are deformed to actual solutions in Section 4, where the other results are also proved.

\section{Geometry of the COMPLEX hyperbolic SPACE}

Here we recall some basic facts about complex hyperbolic geometry. All the material is standard; see the book [11]. 
2.1. Definition. The complex hyperbolic space is described as follows. The complex vector space $\mathbb{C}^{n+1}$ is endowed with the Hermitian form of signature $(n, 1)$.

$$
\left\langle Z, Z^{\prime}\right\rangle=2\left(\bar{z}_{0} z_{n}^{\prime}+\bar{z}_{n} z_{0}^{\prime}\right)+\sum_{k=1}^{n-1} \bar{z}_{k} z_{k}^{\prime},
$$

where $Z=\left(z_{0}, z_{2}, \cdots, z_{n}\right)$ and $Z^{\prime}=\left(z_{0}^{\prime}, z_{2}^{\prime}, \cdots, z_{n}^{\prime}\right)$. The complex hyperbolic space is defined by

$$
\mathbb{C} \mathcal{H}^{n}=\left\{[Z] \in \mathbb{C P}^{n} \quad \mid \quad\langle X, X\rangle<0\right\} .
$$

It is endowed with a Kähler-Einstein metric, called the complex hyperbolic metric, defined as follows: at $[Z] \in \mathbb{C} \mathcal{H}^{n}$ put

$$
g_{Z}^{\mathbb{C H}}(V, V)=4 \frac{\langle Z, Z\rangle\langle V, V\rangle-\langle Z, V\rangle\langle Z, X\rangle}{-\langle Z, Z\rangle^{2}}
$$

for any tangent vector $V \in T_{Z} \mathbb{C}^{n+1}$. Notice that we are using conventions for which the metric has sectional curvature $-1 / \leq K \leq-1 / 4$.

2.2. Dilations and inversions. Consider the family of hyperbolic transformations given by the matrices

$$
H_{\mu}=\left(\begin{array}{ccc}
\frac{1}{\bar{\mu}} & 0 & 0 \\
0 & I_{n-1} & 0 \\
0 & 0 & \mu
\end{array}\right)
$$

for any $\mu \in \mathbb{C}^{*} \backslash\{1\}$.

The points

$$
\zeta^{-}=[0: 0: \cdots: 1], \quad \zeta^{+}=[1: 0: \cdots: 0]
$$

of $\partial_{\infty} \overline{\mathbb{C H}}^{n}$ are the only points of $\overline{\mathbb{C H}}^{n}$ fixed by the isometry $H_{\mu}$.

For $\lambda>0$, we define a hypersurface of $\mathbb{C} \mathcal{H}^{n}$ as

$$
D_{\lambda}=\left\{\left[z_{0}: \cdots: z_{n}\right] \in \mathbb{C H}^{n}|\lambda| z_{0}|=| z_{n} \mid\right\} .
$$

We clearly have

$$
H_{\mu} D_{\lambda}=D_{\lambda|\mu|^{2}} .
$$

The hypersurface $D_{\lambda}$ (a topological disk) splits the hyperbolic space into two connected components, and we have a decomposition

$$
\mathbb{C H}^{n}=B_{\lambda}^{-} \cup D_{\lambda} \cup B_{\lambda}^{+},
$$

where

$$
\begin{aligned}
& B_{\lambda}^{-}=\left\{\left[z_{0}: \cdots: z_{n}\right] \in \mathbb{C H}^{n} \quad|\quad \lambda| z_{0}|<| z_{n} \mid\right\}, \\
& B_{\lambda}^{+}=\left\{\left[z_{0}: \cdots: z_{n}\right] \in \mathbb{C H}^{n} \quad|\quad \lambda| z_{0}|>| z_{n} \mid\right\}
\end{aligned}
$$

are topological balls. The half-ball $B_{\lambda}^{ \pm}$is by construction a neighborhood of the point at infinity $\zeta^{ \pm}$. As $\lambda \rightarrow 0$, the points of $B_{\lambda}^{+}$converge to $\zeta^{+}$in the topology induced by $\mathbb{C P}^{n}$, and, the points of $B_{\lambda}^{-}$converge to $\zeta^{-}$as $\lambda \rightarrow \infty$.

The inversion of $\mathbb{C H}^{n}$,

$$
I_{1}:\left[z_{0}: \cdots: z_{n}\right] \mapsto\left[z_{n}: z_{1}: \cdots: z_{n-1}: z_{0}\right],
$$

is an isometry and it is clearly a holomorphic involution of $\mathbb{C H} \mathcal{H}^{n}$ leaving the disk $D_{1}$ invariant and switching $B_{1}^{+}$and $B_{1}^{-}$. 
Composing with the complex conjugation, we get an antiholomorphic transformation $K_{1}:=\bar{I}_{1}$, i.e.

$$
K_{1}:\left[z_{0}: \cdots: z_{n}\right] \mapsto\left[\bar{z}_{n}: \bar{z}_{1}: \cdots: \bar{z}_{n-1}: \bar{z}_{0}\right],
$$

which is also an isometric involution. The balls $B_{1}^{+}$and $B_{1}^{-}$are exchanged by $K_{1}$, and the disk $D_{1}$ is moreover fixed by $K_{1}$. This transformation will be called a conversion 1

We deduce a family of inversions $I_{\lambda}$ and conversions $K_{\lambda}$ defined by conjugation

$$
I_{\lambda}:=H_{\mu} I_{1} H_{\mu}^{-1}, \quad K_{\lambda}:=\overline{I_{\lambda}},
$$

where $\mu$ is any complex number such that $|\mu|^{2}=\lambda$. We get the explicit formula

$$
\begin{aligned}
I_{\lambda}:\left[z_{0}: \cdots: z_{n}\right] & \mapsto\left[\frac{z_{n}}{\lambda}: z_{1}: \cdots: z_{n-1}: \lambda z_{0}\right], \\
K_{\lambda}:\left[z_{0}: \cdots: z_{n}\right] & \mapsto\left[\frac{\bar{z}_{n}}{\lambda}: \bar{z}_{1}: \cdots: \bar{z}_{n-1}: \lambda \bar{z}_{0}\right] .
\end{aligned}
$$

Again $I_{\lambda}, K_{\lambda}$ are isometric involutions which preserve $D_{\lambda}$ and exchange $B_{\lambda}^{ \pm}$. The inversions are holomorphic, whereas the conversions are antiholomorphic.

Using the isometries $H_{\mu}$ and, say, $I_{\lambda}$, it is clear that the half spaces $B_{\lambda}^{+}$and $B_{\lambda}^{-}$ are all isometric. The disks $D_{\lambda}$ are all isometric as well.

\subsection{The paraboloid model. The function}

$$
f=-\frac{\langle Z, Z\rangle}{4\left|z_{0}\right|^{2}}
$$

is well defined on $\mathbb{C}^{n+1} \backslash\left\{z_{0}=0\right\}$ and $\mathbb{C}^{*}$ invariant. Therefore $f$ can be seen as a smooth function on $\overline{\mathbb{C H}}^{n} \backslash\left\{\zeta^{-}\right\}$and it is a defining function for the boundary $\partial \overline{\mathbb{C H}}^{n} \backslash\left\{\zeta^{-}\right\}$, i.e. $f>0$ on $\mathbb{C} \mathcal{H}^{n}$ and $\partial \overline{\mathbb{C H}}^{n} \backslash\left\{\zeta^{-}\right\}=f^{-1}(0)$.

By the definition of $f$, it is convenient to use the affine coordinates given by fixing $z_{0}=-1$, so that

$$
f=\operatorname{Re}\left(z_{n}\right)-\frac{1}{4}\left(\left|z_{1}\right|^{2}+\cdots+\left|z_{n-1}\right|^{2}\right) .
$$

Thus, we have the model of the Siegel domain

$$
\mathbb{C H}^{n}=\left\{\left(z_{1}, \cdots, z_{n}\right) \in \mathbb{C}^{n} \mid f\left(z_{1}, \cdots, z_{n}\right)>0\right\} .
$$

Notice that $\mathbb{C H}^{n}$ is foliated by paraboloids, namely the level surfaces of $f$

$$
\mathcal{P}_{\alpha}=f^{-1}(\alpha),
$$

for $\alpha>0$ and $\mathcal{P}_{0}$ corresponds to the boundary at infinity of $\mathbb{C} \mathcal{H}^{n}$ minus $\zeta^{-}$(the Heisenberg group). These surfaces are horospheres for the complex hyperbolic metric.

Notice the property

$$
H_{\mu} \mathcal{P}_{\alpha}=\mathcal{P}_{\alpha|\mu|^{2}} .
$$

Hence $H_{\mu} \mathcal{P}_{\alpha}$ converge to the boundary paraboloid as $|\mu| \rightarrow 0$. We can regard the complex hyperbolic space as a stack of hyperboloids using the diffeomorphism

$$
\begin{aligned}
& \mathbb{C H}^{n} \stackrel{\phi}{\longrightarrow}(0, \infty) \times \mathcal{P}_{0}, \\
& \left(z_{1}, \cdots, z_{n}\right) \longmapsto\left(f,\left(z_{1}, \cdots, z_{n-1}, z_{n}-\frac{f}{4}\right)\right) .
\end{aligned}
$$

\footnotetext{
${ }^{1}$ Conversion $=$ con + version, from $\underline{\text { conjugation and inversion. }}$.
} 
This diffeomorphism gives us the horospherical coordinates on $\mathbb{C H}^{n}$ :

$$
\left(f+i v=\bar{z}_{n}-\frac{1}{4}\left(\left|z_{1}\right|^{2}+\cdots+\left|z_{n-1}\right|^{2}\right), W=\left(z_{1}, \cdots, z_{n-1}\right)\right) .
$$

Notice that $\phi$ induces a diffeomorphism between $\mathcal{P}_{\alpha}$ and $\{\alpha\} \times \mathcal{P}_{0}$.

We express the complex hyperbolic metric using the horospherical coordinates

$$
g^{\mathbb{C H}}=\frac{d f^{2}+\eta_{0}^{2}}{f^{2}}+\frac{|d W|^{2}}{f},
$$

where

$$
\eta_{0}=d v+\frac{1}{2} \operatorname{Im}(\bar{W} d W)=d v+\frac{1}{2} \operatorname{Im}\left(\bar{z}_{1} d z_{1}+\cdots+\bar{z}_{n-1} d z_{n-1}\right)
$$

is the standard invariant contact form on the Heisenberg group, and the metric $|d W|^{2}=\left|d z_{1}\right|^{2}+\cdots+\left|d z_{n-1}\right|^{2}$ is obtained from the contact form and the complex structure $J_{0}$ by the formula

$$
|d W|^{2}=d \eta_{0}\left(\cdot, J_{0} \cdot\right)
$$

Finally it is important to note that $-\ln f$ is a potential for the Kähler form $\omega_{0}$ of $\mathbb{C H}^{n}$ :

$$
\omega_{0}=-d d^{C} \ln f=-2 i \partial \bar{\partial} \ln f .
$$

\section{Pregluing}

We have reviewed the basics of the complex hyperbolic space, and we can define new complex hyperbolic manifolds via Klein construction. We are now on firm ground to introduce the gluing theory inspired by this construction.

3.1. Annulus near a point at infinity. Let $X$ be a complex manifold endowed with a complex hyperbolic metric $g$. In other words, $X$ is a quotient of $\mathbb{C H}^{n}$ by a group of isometries. Assume in addition that the induced metric on $X$ is ACH. Pick a point $p$ at infinity. Since the metric is hyperbolic, the point $p$ has a neighborhood $B_{1} \subset X$ which is an isometric copy of $B_{1}^{+} \subset \mathbb{C H}^{n}$, and $p$ is identified to $\zeta_{+}$(cf. Section 2.2), via an isometry

$$
\psi: B_{1} \longrightarrow B_{1}^{+} \text {. }
$$

Since $B_{\lambda_{0}}^{+} \subset B_{\lambda_{1}}^{+}$for $0<\lambda_{0}<\lambda_{1}$, we can define a neighborhood $B_{\lambda} \subset X$ of $p$ by

$$
B_{\lambda}:=\psi^{-1}\left(B_{\lambda}^{+}\right) \text {, for } 0<\lambda<1 .
$$

Given a pair $\underline{\lambda}=\left(\lambda_{0}, \lambda_{1}\right)$ with $0<\lambda_{0}<\lambda_{1}$, we define the annulus

$$
U_{\underline{\lambda}}:=B_{\lambda_{1}}^{+} \backslash B_{\lambda_{0}}^{+} .
$$

Notice that the annulus $U_{\underline{\lambda}}$ has boundary $D_{\lambda_{0}}$. Accordingly we define (provided $\left.\lambda_{j} \leq 1\right)$

$$
V_{\underline{\lambda}}:=B_{\lambda_{1}} \backslash B_{\lambda_{0}}=\psi^{-1}\left(U_{\underline{\lambda}}\right) \subset X .
$$

Lemma 3.1.1. Let $\underline{\lambda}^{k}=\left(\lambda_{0}^{k}, \lambda_{1}^{k}\right)(k=0,1)$ be two pairs of positive numbers such that $\lambda_{0}^{k}<\lambda_{1}^{k}(k=0,1)$ and

$$
\lambda_{0}^{0} \lambda_{1}^{1}=\lambda_{0}^{1} \lambda_{1}^{0}
$$

Then the manifold with boundary $U_{\underline{\lambda}^{k}}$ are isometric for $k=1,2$. Moreover,

$$
H_{\mu}: U_{\underline{\lambda}^{0}} \rightarrow U_{\underline{\lambda}^{1}}
$$


is a particular isometry for any $\mu \in \mathbb{C}$ such that

$$
|\mu|^{2}=\frac{\lambda_{0}^{1}}{\lambda_{0}^{0}}=\frac{\lambda_{1}^{1}}{\lambda_{1}^{0}}
$$

Proof. The proof is clear using a hyperbolic isometry as in the lemma.

Lemma 3.1.2. There exists an inversion $I_{\underline{\lambda}}$ (resp. a conversion $K_{\underline{\lambda}}$ ) which is an isometry of $\bar{U}_{\underline{\lambda}}$ and exchanges the boundary components $D_{\lambda_{0}}$ and $D_{\lambda_{1}}$. In addition the disk $D_{\sqrt{\lambda_{0} \lambda_{1}}}$ is preserved by this transformation and also if the function $f$ is restricted to this disk.

Proof. By Lemma 3.1.1 there is an isometry

$$
H_{\mu}: U_{\underline{\lambda}} \rightarrow U_{\underline{\lambda}^{\prime}},
$$

where

$$
\underline{\lambda}^{\prime}=\left(\sqrt{\frac{\lambda_{0}}{\lambda_{1}}}, \sqrt{\frac{\lambda_{1}}{\lambda_{0}}}\right), \quad|\mu|^{2}=\left(\lambda_{0} \lambda_{1}\right)^{-1 / 2} .
$$

Then, the inversion $I_{1}$ preserves the annulus $\bar{U}_{\underline{\lambda}^{\prime}}$, the disk $D_{1}$ and exchanges the boundary components as wanted. We have $H_{\mu}^{*} f=|\mu|^{f}$, and $\left.f\right|_{D_{1}}$ is invariant under $I_{1}$. In conclusion, the inversion $I_{\sqrt{\lambda_{0} \lambda_{1}}}$ answers the lemma. We deduce that the conversion $K_{\sqrt{\lambda_{0} \lambda_{1}}}=\bar{I}_{\sqrt{\lambda_{0} \lambda_{1}}}$ answers the lemma for the case of a conversion.

3.2. ACH metrics. Here we give a more precise technical definition of an ACH metric.

3.2.1. Definition. As in the Introduction, $(Y, J)$ is a CR manifold, with a $\mathrm{CR}$ structure defined along a contact distribution with a contact form $\eta$. The $\mathrm{CR}$ structure is assumed to be strictly pseudoconvex in the sense that $\gamma(\cdot, \cdot)=d \eta(\cdot, J \cdot)$ defines a Hermitian metric along the contact distribution. The manifold $\bar{X}$ has boundary $Y$, and we choose a defining function $u$ of the boundary and identify a collar neighborhood of $Y$ with $Y \times[0, T)$. Then on this collar neighborhood we have a model metric

$$
g_{0}=\frac{d u^{2}+\eta^{2}}{u^{2}}+\frac{\gamma}{u}
$$

Also we will often use the weight function $w=\sqrt{u}$.

We say that a metric $g$ on $X$ is $\mathrm{ACH}$, with conformal infinity $J$, if near the boundary one has

$$
g=g_{0}+\kappa,
$$

where $\kappa$ is a symmetric 2-tensor, such that $|\kappa|=O\left(w^{\delta_{0}}\right)$, and more generally all derivatives satisfy $\left|\nabla^{k} \kappa\right|=O\left(w^{\delta_{0}}\right)$ for a weight $\delta_{0} \leq 1$ which will be fixed thorough the paper. (Here, all the norms and derivatives are taken with respect to the metric $g_{0}$.) Actually, we shall use the convenient choice $\delta_{0}=1$, because in an asymptotic expansion of an Einstein metric $g$ with conformal infinity $J$, the first correction may occur at order 1 only. This is made precise in the following statement.

Proposition 3.2.2. Suppose that $g$ is an ACH Einstein metric with conformal infinity $J$ for some weight $\delta<1$. Then, by a diffeomorphism of $X$ inducing the identity on $Y$, one can put $g$ in a gauge where $g=g_{0}+\kappa$ and $\left|\nabla^{k} \kappa\right|=O(w)$ for all $k \geq 0$. 
Proof. In dimension 4, a much stronger asymptotic expansion is constructed in 3 , Section 5], and one can take $\delta_{0}=2$. In higher dimension, one must take only $\delta_{0}=1$, because the Nijenhuis tensor of $J$ is a first order invariant and occurs in the correction of $g_{0}$ at order 1 .

We shall not write the proof of the proposition, which is simpler that the 4dimensional case proved in [3]. It suffices to put $g$ in a Bianchi gauge with respect to $g_{0}$ as in [3. Lemma 4.1], and then to analyze its regularity.

Example 3.2.3. An important case of ACH metrics is when $\bar{X}$ is a complex manifold with strictly pseudoconvex boundary $Y$. Choose any defining function $u$ of the boundary. Then one can generalize (2.8) in the following way: the formula

$$
\omega=-d d^{C} \ln u
$$

defines in a neighborhood of the boundary the Kähler form of an ACH metric on $X$, with conformal infinity the natural CR structure induced on $Y$. More precisely, choosing on $Y$ the contact form $\eta=-d^{C} u$ and the metric $\gamma=d \eta(\cdot, J \cdot)$, the metric with Kähler form (3.2) satisfies $g=\frac{d u^{2}+\eta^{2}}{u^{2}}+\frac{\gamma}{u}+O(u)$. The metric is KählerEinstein if $u$ satisfies Fefferman's equation [10]:

$$
\operatorname{det}\left(\begin{array}{cc}
u & u_{\bar{k}} \\
u_{j} & u_{j \bar{k}}
\end{array}\right)=\left(-\frac{1}{4}\right)^{n} .
$$

On the other hand, any Kähler ACH metric $\omega$ on $X$ can be written locally near a point of the boundary as deriving from a potential with the same leading term: $\omega=d d^{C}\left(-\ln f+O\left(w^{\delta_{0}}\right)\right)$.

3.3. Standardization of the metric near infinity. In this section, we modify slightly an ACH Einstein metric near a point a infinity, so that the metric is complex hyperbolic. We show that the resulting metric is not far from being Einstein.

3.3.1. The contact structure. Pick a point $p$ in $Y$. Since contact structure has no local invariants, there exists a contactomorphism $\psi: W_{p} \rightarrow W_{\zeta^{+}}$identifying a neighborhood $W_{p} \subset Y$ of $p$ to a neighborhood $W_{\zeta_{+}}$of $\zeta_{+}$in the Heisenberg group $\mathcal{P}_{0}$, such that $\psi(p)=\zeta^{+}$. The contact distribution is preserved by $\psi$; hence

$$
\psi_{*} \eta=h \eta_{0}
$$

for a certain nonvanishing function $h$. Replacing the contact structure $\eta$ by $h^{-1} \eta$ in a neighborhood of $p$, we can assume $h=1$. Then we extend $\psi$ to a diffeomorphism $\psi$ between collar neighborhoods of $W_{p}$ and $W_{\zeta^{+}}$given by

$$
\Psi(u, y)=(u, \psi(y)) .
$$

It follows from the definition that

$$
\Psi_{*} \hat{g}=\frac{d u^{2}+\eta_{0}^{2}}{u^{2}}+\frac{\psi_{*} \gamma}{u} .
$$

Hence the metric of $\bar{X}$, transported by $\Psi$ to the upper half-space (with horospherical coordinates), has the form

$$
\mathrm{g}:=\Psi_{*} g=\frac{d u^{2}+\eta_{0}^{2}}{u^{2}}+\frac{\gamma_{1}}{u}+\kappa,
$$

where $\kappa$ is a symmetric 2-tensor on $\mathbb{C H} \mathcal{H}^{n}$ such that $w^{-1} \kappa$ and all its derivatives are bounded with respect to $g^{\mathbb{C H}}$. Moreover $\gamma_{1}=d \eta_{0}\left(\cdot, J_{1} \cdot\right)$ for the compatible almost 
complex structure $J_{1}=\psi_{*} J$ defined along $\xi_{0}=\operatorname{ker} \eta_{0}$ in $\mathcal{P}_{0}$, and we can always assume that the contactomorphism $\psi$ is chosen so that

$$
J_{1}=J_{0} \text { at } \zeta^{+} .
$$

3.3.2. Approximation for the almost complex structure. Let $\chi(s)$ be a smooth nonnegative increasing function such that $\chi(s)=0$ for $s \leq \frac{1}{3}$ and $\chi(s)=1$ for $s \geq \frac{2}{3}$. Given a pair of numbers $\underline{\tau}=\left(\tau_{0}, \tau_{1}\right)$ with $0<\tau_{0}<\tau_{1}$, we define the cut-off function

$$
\varphi_{\underline{\tau}}(x)=\chi\left(\frac{x-\tau_{0}}{\tau_{1}-\tau_{0}}\right),
$$

and we deduce the function

$$
\chi_{\underline{\underline{\tau}}}=\varphi_{\underline{\underline{\tau}}}\left(\left|\frac{z_{n}}{z_{0}}\right|\right)
$$

on $\mathbb{C} \mathcal{H}^{n}$. By definition $\chi_{\underline{\underline{\tau}}}=0$ in $B_{\tau_{0}}^{+}$and $\chi_{\underline{\underline{\tau}}}=1$ outside $B_{\tau_{1}}^{+}$. Notice that if $\left(z_{1}, \cdots, z_{n}\right) \in \mathcal{P}_{0}$, then $\left(x z_{1}, \cdots, x z_{n-1}, x^{2} z_{n}\right)$ is also in $\mathcal{P}_{0}$. Then we can define

$$
J_{\underline{\tau}}\left(z_{1}, \cdots, z_{n}\right):=J_{1}\left(\chi_{\underline{\tau}} z_{1}, \cdots, \chi_{\underline{\tau}} z_{n-1}, \chi_{\underline{\tau}}^{2} z_{n}\right) .
$$

Notice that $J_{1}$ and $J_{0}$ are independent of $f$ (or $t$ ). Now the family of almost complex structures $J_{\underline{\tau}}$ also depends on $f$. In particular, because of the condition (3.3), $J_{\underline{\tau}}$ is equal to $\bar{J}_{0}$ inside $B_{\tau_{0}}^{+}$and $J_{\underline{\tau}}$ is equal to $J_{1}$ outside $B_{\tau_{1}}^{+}$. We define a family of Carnot-Carathéodory metrics

$$
\gamma_{\underline{\tau}}=d \eta_{0}\left(\cdot, J_{\underline{\tau}}\right)
$$

and the metric $\gamma_{\underline{\underline{\tau}}}$ is constructed in such a way that it is equal to $\gamma_{0}$ in $B_{\tau_{0}}^{+}$and $\gamma_{1}$ outside $B_{\tau_{1}}^{+}$.

Eventually, we can define the Riemannian metrics on $\mathbb{C H}^{n}$ :

$$
\mathrm{g}_{\underline{\underline{\tau}}}=\frac{d u^{2}+\eta_{0}^{2}}{u^{2}}+\frac{\gamma_{\underline{\tau}}}{u}+\chi_{\underline{\underline{\tau}}} \kappa .
$$

The metric $g_{\underline{\tau}}$ is equal to the complex hyperbolic metric in $B_{\tau_{0}}^{+}$(this is the locus where we will apply the Klein construction later). Outside $B_{\tau_{1}}^{+}$it is equal to the original metric $\mathrm{g}$. Hence the metric $\Psi^{*} \mathrm{~g}_{\underline{\tau}}$ on $X$ can be extended using the original metric $g$ outside $B_{\tau_{1}}$. The resulting metric is denoted $g_{\underline{\tau}}$. We expect $g_{\underline{\tau}}$ to be a very good approximation of an Einstein metric, in a sense that will be clarified in the next section.

3.3.3. Integrable case. Here we consider the case where $\bar{X}$ is a complex manifold with boundary and where the metric $g$ on $X$ is Kähler-Einstein. We want to perform the same operation as in (3.4), but remaining in the category of integrable complex structures and Kähler metrics, so we need a refined method. There are two steps: gluing the complex structures and then the metrics. Therefore we need to fix an intermediate $\left.\tau_{2} \in\right] \tau_{0}, \tau_{1}\left[\right.$, for example $\tau_{2}=\sqrt{\tau_{0} \tau_{1}}$, and we set $\underline{\tau}^{\prime}=\left(\tau_{0}, \tau_{2}\right)$ and $\underline{\tau}^{\prime \prime}=\left(\tau_{2}, \tau_{1}\right)$.

We choose complex coordinates $z=\left(z_{i}\right)$ near the point $p$ in $\bar{X}$, so that $Y$ is given by a defining function $u(z)$. Using the normal form of Chern and Moser [7], we can suppose that

$$
u(z)=f(z)+O\left(|W|^{4}\right),
$$


where $f(z)=\operatorname{Re} z_{n}-\frac{1}{4}|W|^{2}$ is the defining function for the half-space model. Actually if the boundary $Y$ is not 3-dimensional, then one can obtain

$$
u(z)=f(z)+O\left(|W|^{6}\right) .
$$

Now, instead of gluing the almost complex structures, we glue the defining functions of $\bar{X}$ and $\mathbb{C} \mathcal{H}^{n}$ in the normal complex coordinates, choosing

$$
u_{\underline{\tau}}=\left(1-\chi_{\underline{\tau}^{\prime}}\right) f+\chi_{\underline{\underline{\prime}}^{\prime}} u \text {. }
$$

Still in the coordinates $\left(z_{i}\right)$, the domains $\bar{X}_{\underline{\underline{\tau}}}:=\left\{u_{\underline{\underline{\tau}}} \geq 0\right\}$ give us a family of complex domains coinciding with the Siegel domain in $B_{\tau_{0}}^{+}$and with $\bar{X}$ outside $B_{\tau_{2}}^{+} \subset B_{\tau_{1}}^{+}$, and there is a natural family of integrable complex structures $J_{\underline{\tau}}$ on $\bar{X}_{\underline{\tau}}$.

We now wish to define a Kähler metric on $\left(\bar{X}, J_{\tau}\right)$ which coincides with the complex hyperbolic metric on $B_{\tau_{0}}^{+}$and with the metric of $X$ outside $B_{\tau_{1}}^{+}$. First remember from (2.8) that the function $\varphi_{0}=-\ln f$ is a potential for the complex hyperbolic metric. On the other hand, the metric $g$ on $X$ (seen in the same complex coordinates) admits a local potential $\varphi=-\ln u+O(w)$; see Example 3.2.3. The solution of the problem is therefore simple: again by Example 3.2.3, the function $-\ln u_{\tau}$ is a potential for a local Kähler metric on $X_{\tau}$, so we can consider the modified potential

$$
\varphi_{\underline{\underline{\tau}}}=-\ln u_{\underline{\underline{\tau}}}+\chi_{\underline{\tau}^{\prime \prime}}(\varphi+\ln u),
$$

which coincides with $\varphi_{0}$ in $B_{\tau_{0}}^{+}$and is equal to $\varphi$ outside $B_{\tau_{1}}^{+}$. This potential defines an $\mathrm{ACH}$ Kähler metric on $\left(X, J_{\underline{\tau}}\right)$ by

$$
\omega_{\underline{\tau}}=d d_{J_{\underline{\tau}}}^{C} \varphi_{\underline{\tau}}
$$

This metric coincides with the complex hyperbolic metric in $B_{\tau_{0}}^{+}$and with $g$ outside $B_{\tau_{1}}^{+}$.

3.4. Estimates. The perturbed metrics $\mathrm{g}_{\tau}$ are not Einstein any more. However they are good approximate Einstein metrics in a sense made precise in the following proposition.

Proposition 3.4.1. Let $c$ be a constant with $c>1$. There exists a constant $C>0$, depending only on the metric $g$ and $c$, such that for any pair of numbers $\underline{\tau}=\left(\tau_{0}, \tau_{1}\right)$ with $0<\tau_{0}<\tau_{1}$ and $c \tau_{0} \leq \tau_{1}$, the metric $\mathrm{g}_{\underline{\underline{\tau}}}$ verifies

$$
\left|\mathrm{g}_{\underline{\underline{\tau}}}-g^{\mathbb{C H}}\right| \leq C \tau_{1}^{1 / 2} \quad \text { and } \quad\left|\operatorname{Ric}^{\mathrm{g}_{\underline{I}}}+\frac{n+1}{2} \mathrm{~g}_{\underline{\underline{\tau}}}\right| \leq C w
$$

on the annulus $U_{\underline{\underline{\tau}}}$ (the norm being taken w.r.t. the metric $g^{\mathbb{C H}}$ ). A similar statement holds for derivatives of higher order.

The construction carried out in Section 3.3.2 uses annuli $U_{\underline{\tau}}$ which get smaller and smaller in the sense that $\underline{\tau}=\left(\tau_{0}, \tau_{1}\right)$ with $\tau_{j} \rightarrow 0$. It is convenient for computations to "resize" $U_{\tau}$ : pick a transformation $H_{\mu}$, for some complex number $\mu$ such that $|\mu|^{2}=\tau_{1}$, for instance $H_{\sqrt{\tau_{1}}}$. Then

$$
H_{\mu}: U_{\underline{\tau}^{\prime}} \rightarrow U_{\underline{\tau}}, \quad \text { where } \quad \underline{\tau}^{\prime}:=\left(\tau_{0} / \tau_{1}, 1\right) .
$$

Notice that the assumption of Proposition 3.4 .1 means that $\tau_{0} / \tau_{1}$ is bounded away from 1 , so that the annulus $U_{{\underline{\tau^{\prime}}}^{\prime}}$ cannot be too "thin". 
The hyperbolic transformation acts in the half-space model by scaling the coordinates:

$$
H_{\sqrt{\tau_{1}}}\left(z_{1}, \cdots, z_{n}\right)=\left(\sqrt{\tau_{1}} z_{1}, \cdots, \sqrt{\tau_{1}} z_{n-1}, \tau_{1} z_{n}\right)
$$

Hence the hyperbolic transformation acts on the paraboloid at infinity $\mathcal{P}_{0}$ by scaling the coordinates as above. The boundary at infinity of $B_{\tau}^{+}$is given by $\partial_{\infty} B_{\tau}^{+}:=$ $\bar{B}_{\tau}^{+} \cap \mathcal{P}_{0}$, which is the open set

$$
\partial_{\infty} B_{\tau}^{+}=\left\{\left(z_{1}, \cdots, z_{n}\right) \mid f\left(z_{1}, \cdots, z_{n}\right)=0 \text { and }\left|z_{n}\right|<\tau\right\} .
$$

In particular

$$
\left(z_{1}, \cdots, z_{n}\right) \in \partial_{\infty} B_{\tau}^{+} \Rightarrow\left|z_{n}\right|<\tau \text { and }\left|z_{j}\right|<2 \sqrt{\tau} \text { for } j=1, \cdots, n-1 .
$$

From this observation, we deduce the following lemma:

Lemma 3.4.2. Let $J_{1}$ be an almost complex structure defined on the standard contact distribution $\xi_{0}$ of $\mathcal{P}_{0}$ and let $J_{0}$ be the standard $C R$ structure. Assume that $J_{0}=J_{1}$ at $\zeta_{+}$. Then there are constants $C_{k}>0$ such that for every $0<\tau<1$

$$
\left|T_{\tau}\right| \leq C_{0} \tau^{1 / 2} \text { in } \partial_{\infty} B_{1}^{+}
$$

where

$$
T_{\tau}=H_{\sqrt{\tau}}^{*} J_{1}-J_{0}
$$

and the norm is taken w.r.t. the standard metric induced by $\mathbb{C}^{n}$. In the case of derivatives, we have

$$
\left|\nabla^{k} T_{\tau}\right| \leq C_{k} \tau^{k / 2} \text { in } \partial_{\infty} B_{1}^{+}
$$

Proof. Notice that the standard CR-structure is invariant under hyperbolic isometries. In particular $H_{\sqrt{\tau}}^{*} J_{0}=J_{0}$. So the first part of the lemma follows from the fact that $A_{\tau}=0$ at $\zeta_{+}$and (3.9). The second part is a consequence of the fact that the derivatives of $J_{1}-J_{0}$ are bounded in a neighborhood of $\zeta_{+}$and the Leibniz rule applied to $T_{\tau}$ in view of (3.8).

The above lemma can be readily generalized as follows:

Lemma 3.4.3. Let $J_{\tau}$ be the family of almost complex structures defined in Section 3.4. Suppose that $c \tau_{0} \leq \tau_{1}$ as in Proposition 3.4.1. Then there are constants $C_{k}>0$ (independent of $\underline{\tau}$ ) such that

$$
\left|T_{\underline{\tau}}\right| \leq C_{0} \tau_{1}^{1 / 2}, \quad \text { and }\left|\nabla^{k} T_{\underline{\tau}}\right| \leq C_{k} \tau_{1}^{k / 2} \text { for } k \geq 1
$$

in $B_{1}^{+}$, where

$$
T_{\underline{\tau}}=H_{\sqrt{\tau_{1}}}^{*} J_{\underline{\tau}}-J_{0}
$$

and the norm is taken w.r.t. the standard metric induced by $\mathbb{C}^{n}$.

Proof of Proposition 3.4.1. First note that the term $\kappa$ gives a perturbation which is uniformly bounded by $w$, so we can assume that $\kappa=0$ and deal with the perturbation of $\gamma_{0}$.

The first part of the proposition is a direct consequence of Lemma 3.4.3. If we pull back the metric $\mathrm{g}_{\underline{\mathcal{\tau}}}$ on $B_{1}^{+}$thanks to a hyperbolic transformation, the Carnot-Carathéodory metric is commensurate with the standard $\gamma_{0}$ using (3.10). We deduce that $\left|H_{\sqrt{\tau_{1}}}^{*} \mathrm{~g}_{\underline{\tau}}-g^{\mathbb{C H}}\right|$ is controlled by $\tau_{1}^{1 / 2}$. Derivatives of order $k$ are controlled by $\tau_{1}^{k / 2}$. Since $H_{\mu}$ is an isometry of $g^{\mathbb{C H}}$, the same control holds for $\mathrm{g}_{\underline{\tau}}-g^{\mathbb{C H}}$. 
We consider the vector fields

$$
\begin{aligned}
& x_{0}=u \partial_{u}, \\
& x_{1}=R, \\
& x_{j} \in \xi_{0},
\end{aligned}
$$

where $R$ is the Reeb vector field defined by $\eta_{0}(R)=1$ and $\iota_{R} d \eta_{0}=0$, and $X_{j}$ is an orthonormal basis of $\xi_{0}$ with respect to the metric $\gamma_{0}$ for $j=2, \cdots, 2 n-2$. Then, we have an orthonormal frame for $g^{\mathbb{C H}}$ given by

$$
y_{0}=x_{0}, \quad y_{1}=u x_{1} \quad \text { and } \quad y_{j}=\sqrt{u} x_{j} \quad \text { for } j \geq 2 .
$$

According to Lemma 3.4.3 there exist perturbations $\tilde{x}_{j}$ of the vector fields $x_{j}$ for $j \geq 2$, so that $\tilde{X}_{j}$ is an orthonormal frame for $\gamma_{\underline{\tau}}$ and the pointwise norm of $H_{\sqrt{\tau_{1}}}^{*}\left(\tilde{x}_{j}-x_{j}\right)$ is controlled by $\tau_{1}^{1 / 2}$. Using $x_{0}, x_{1}, \overline{\tilde{x}}_{2}, \cdots, \tilde{x}_{2 n-2}$, we define the orthonormal frame $\tilde{y}_{j}$ for $\underline{g}_{\underline{\tau}}$ similarly to $y_{j}$.

Lemma 3.4.4. We have the following identities:

$$
\begin{aligned}
& {\left[\tilde{y}_{0}, \tilde{y}_{1}\right]=-2 \tilde{y}_{1},} \\
& {\left[\tilde{y}_{0}, \tilde{y}_{j}\right]=-\tilde{y}_{j}+O(w) \text { for } j \geq 2,} \\
& {\left[\tilde{y}_{1}, \tilde{y}_{j}\right]=O(w) \text { for } j \geq 1,} \\
& {\left[\tilde{y}_{i}, \tilde{y}_{j}\right]=d \eta_{0}\left(\tilde{x}_{i}, \tilde{x}_{j}\right) \tilde{y}_{1}+O(w) \text { for } i, j \geq 2,}
\end{aligned}
$$

where $O(w)$ is a tensor which decays as $w$ w.r.t. the metric $\mathrm{g}_{\underline{\underline{\tau}}}$ and involves a uniform constant, independent of $\tau_{1}$.

Proof. The idea is the following: pull back the metric $\mathrm{g}_{\tau}$ and all the vector fields using the hyperbolic isometry $H=H_{\sqrt{\tau_{1}}}$. For $j \geq 2$, we have

$$
\begin{aligned}
{\left[H^{*} y_{0}, H^{*}\left(\tilde{y}_{j}-y_{j}\right)\right] } & =\left[u \partial_{u}, w H^{*}\left(\tilde{X}_{j}-X_{j}\right)\right] \\
& =\frac{1}{2} w H^{*}\left(\tilde{X}_{j}-X_{j}\right)+w\left[u \partial_{u}, H^{*}\left(\tilde{X}_{j}-X_{j}\right)\right] .
\end{aligned}
$$

Using the control by $\sqrt{\tau_{1}}$ on $H^{*}\left(\tilde{X}_{j}-X_{j}\right)$, we deduce that

$$
\left[H^{*} y_{0}, H^{*}\left(\tilde{y}_{j}-y_{j}\right)\right]=O\left(\sqrt{\tau_{1}} w\right) \text {. }
$$

But $\left(\sqrt{\tau_{1}} w\right) \circ H=w$, hence we have the control

$$
\left[y_{0},\left(\tilde{y}_{j}-y_{j}\right)\right]=O(w) \text {. }
$$

Since $\left[y_{0}, y_{j}\right]=-y_{j}$ for $j \geq 2$, we deduce the identity (3.12). The other identities are proved in the same manner.

The proposition is now deduced from the above lemma using the same computation as in [2, Section I.1.B].

3.5. The Klein construction. Given an ACH Einstein manifold $(X, g)$, we pick two points $p_{0}, p_{1}$ which belong to the boundary $Y=\partial \bar{X}$. A modification of the metric in a neighborhood of one point $p \in Y$ was defined in Section 3.3. We perform the same operation near both points $p_{0}$ and $p_{1}$, and call the resulting metric $g_{\underline{\tau}}$ as well. The parameters of the construction at $p_{j}$ are denoted $\left(\tau_{0}^{j}, \tau_{1}^{j}\right)$. We call $B_{\tau}\left(p_{j}\right) \subset X$ the neighborhood of $p_{j}$ and $V_{\left(\tau_{0}^{j}, \tau_{1}^{j}\right)}\left(p_{j}\right)$ the annular regions 
near $p_{j}$ (defined as in Section 3.3). By construction, the restriction of $g_{\underline{\underline{\tau}}}$ to $B_{\tau_{0}^{j}}\left(p_{j}\right)$ is isometric to the neighborhood $B_{\tau_{0}^{j}}^{+} \subset \mathbb{C} \mathcal{H}^{n}$ of $\zeta_{+}$.

From now on, we fix arbitrarily (for instance)

$$
\tau_{0}^{j}=\tau_{1}^{j} / 2,
$$

so that we can apply Proposition 3.4 .1 to the metrics $g_{\underline{\underline{\tau}}}$. We choose additional parameters $\lambda_{0}^{j}$ and $\lambda_{1}^{j}$ such that

$$
0<\lambda_{0}^{j}<\lambda_{1}^{j}<\tau_{0}^{j}<\tau_{1}^{j}<1
$$

and

$$
K^{2}=\frac{\lambda_{1}^{0}}{\lambda_{0}^{0}}=\frac{\lambda_{1}^{1}}{\lambda_{0}^{1}}
$$

The condition (3.16) implies that the restriction of $g_{\underline{\underline{\tau}}}$ to the annulus $V_{\left(\lambda_{0}^{j}, \lambda_{1}^{j}\right)}\left(p_{j}\right)$ is an isometric copy of $U_{\left(\lambda_{0}^{j}, \lambda_{1}^{j}\right)}\left(p_{j}\right) \subset \mathbb{C} \mathcal{H}^{2}$. Moreover, the condition (3.17) ensures that the annuli $V_{\left(\lambda_{0}^{j}, \lambda_{1}^{j}\right)}\left(p_{j}\right)$ are isometric for $j=0,1$. The Klein construction close to $p_{0}$ and $p_{1}$ consists of the following operation: we consider the manifold with boundary

$$
\widetilde{X}=X \backslash\left(B_{\lambda_{0}^{0}}\left(p_{0}\right) \cup B_{\lambda_{0}^{1}}\left(p_{1}\right)\right) .
$$

A neighborhood of the boundary of $\widetilde{X}$ is given by the annuli $\bar{V}_{\left(\lambda_{0}^{j}, \lambda_{1}^{j}\right)}\left(p_{j}\right)$, which are identified via an inversion $I$. Then, we define the closed manifold

$$
X_{\underline{\underline{x}}}^{\sharp}=\widetilde{X} / I
$$

and call $g_{\underline{\tau}}^{\sharp}$ the resulting metric on $X_{\underline{\underline{\tau}}}^{\sharp}$. The boundary $Y^{\sharp}:=\partial \bar{X}_{\underline{\tau}}^{\sharp}$ has an induced CR structure $J_{\underline{\underline{\tau}}}^{\sharp}$ since the identification $I$ is holomorphic. Notice that the metric $g_{\underline{\underline{\tau}}}^{\sharp}$ is by construction $\mathrm{ACH}$ with conformal infinity $\left(Y^{\sharp}, J_{\underline{\underline{\tau}}}^{\sharp}\right)$.

A similar construction can be done by using a conversion $K$ rather than an inversion, and we put

$$
X_{\underline{\tau}}^{b}=\tilde{X} / K .
$$

Notice that the gluing parameter $\underline{\tau}$ now consists of 8 variables $\left(\lambda_{i}^{j}, \tau_{i}^{j}\right)_{i, j=0,1}$ verifying the constraints (3.15), (3.16) and (3.17). It is convenient to use the notation

$$
\underline{\tau} \rightarrow 0
$$

for a family of parameters $\underline{\tau}$ such that all coefficients $\lambda_{i}^{j} \rightarrow 0, \tau_{i}^{j} \rightarrow 0$. We illustrate our construction in Figure 1 .

The construction of $X_{\tau}^{\sharp}$ is represented in a schematic way. The neighborhoods of $p_{0}$ and $p_{1}$ are pictured on the left and on the right. On each side, the striped annulus corresponds to the parameters $\lambda_{i}^{j}$; this is the region where the metric $g$ is altered. Inside the striped shell the metric is isometric to the complex hyperbolic metric. The gray part is the neighborhood of $p$ which is deleted. The squared part is isometric to the region of $\mathbb{C H}^{n}$ pictured in the middle, which represents the handle addition, and comes with an isometric inversion as suggested by the line.

Remark 3.5.1. It will be important in the gluing technique, in particular for Proposition 4.3.2, to impose that the ratio $\lambda_{1}^{j} / \lambda_{0}^{j}$ goes to infinity. It means that in the 

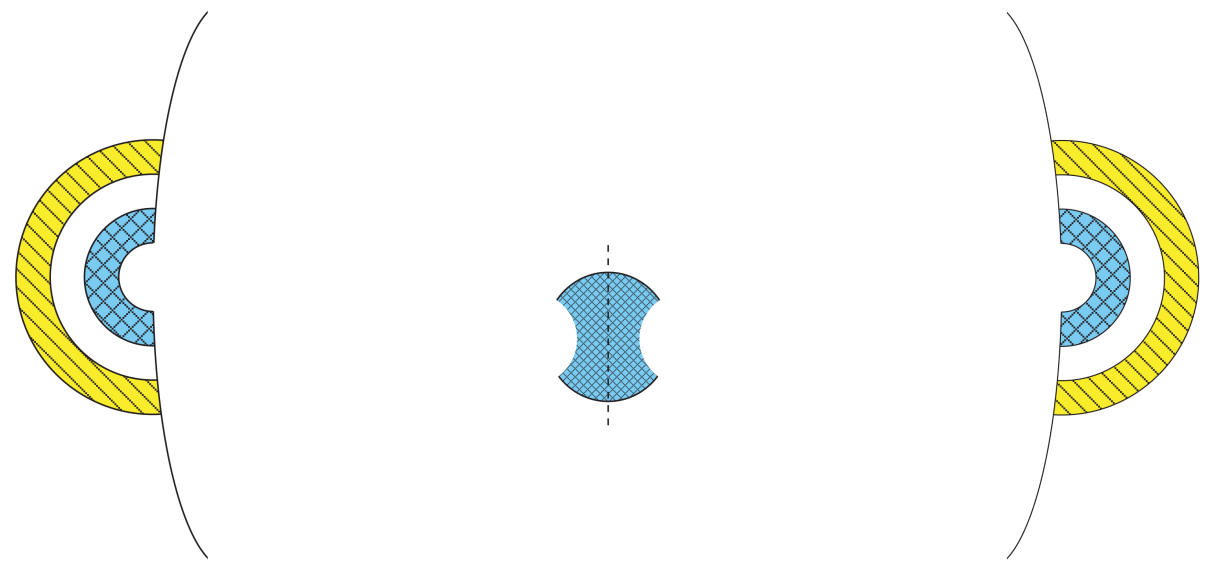

FiguRE 1. 1-handle attachment

above picture, the squared annulus is close to be the entire ball, namely the complex hyperbolic plane itself. So we impose from now on

$$
\lambda_{0}^{j}=\left(\lambda_{1}^{j}\right)^{2} .
$$

The manifold $X_{\underline{\tau}}^{\sharp}$ contains essentially two pieces:

- $Z_{\underline{\tau}} \subset X_{\underline{\tau}}^{\sharp}$ is the closed set defined by $X \backslash\left(B_{\lambda_{1}^{0}}\left(p_{0}\right) \cup B_{\lambda_{1}^{1}}\left(p_{1}\right)\right)$,

- $W_{\underline{\tau}}$ is given by the identified copies of the annuli $\bar{V}_{\left(\lambda_{0}^{j}, \lambda_{1}^{j}\right)}\left(p_{j}\right)$.

In other words, $Z_{\tau}$ is the set of points not in the squared region, whereas $W_{\underline{\tau}}$ is the squared region. Notice that we can consider either $Z_{\underline{\tau}} \subset X_{\underline{\tau}}^{\sharp}$ or $Z_{\underline{\tau}} \in X$.

Remark 3.5.2. Topologically, $X^{\sharp}$ is obtained by a 1-handle addition and does not depend on the choice of parameters. However, the metric $g_{\tau}^{\sharp}$ depends on the ratio $\lambda_{0}^{0} / \lambda_{0}^{1}$. Moreover, there is an extra $S^{1} \times U(n-1)$-freedom for identifying the annuli. To see that, one needs to replace the identity block $I_{n-1}$ in $H_{\mu}$ by a unitary matrix an $\mu$ with $\mu e^{i \theta}$.

Remark 3.5.3. If $X$ is complex hyperbolic and $\mathrm{ACH}$, the Klein construction $X_{\tau}^{\sharp}$ produces another $\mathrm{ACH}$ complex hyperbolic manifold, whereas $X_{\tau}^{b}$ produces an $\mathrm{ACH}$ locally complex hyperbolic manifold, in the sense that the compatible complex structure is only locally defined.

The geometry of the metrics $g_{\underline{工}}^{\sharp}$ is uniform, in the following sense.

Lemma 3.5.4. The injectivity radius of the metrics $g_{\underline{I}}^{\sharp}$ are bounded below by a constant $\rho>0$ which does not depend on $\underline{\tau}$. Moreover, one can cover $X_{\underline{\underline{\tau}}}$ by balls of radius $\rho$ such that, in each ball, one can write $g_{\underline{I}}^{\sharp}=\left(g_{\underline{I}}^{\sharp}\right)_{i j}$ with

$$
\frac{1}{c_{0}} \sum\left(d x_{i}\right)^{2} \leq g_{\underline{\tau}}^{\sharp} \leq c_{0} \sum\left(d x_{i}\right)^{2}
$$

and

$$
\left|\nabla^{k} g_{\underline{\underline{I}}}^{\sharp}\right| \leq c_{k},
$$

and the constants $c_{i}$ do not depend on the ball or on $\underline{\tau}$. 
Proof. If we do not take off the two balls about $p_{0}$ and $p_{1}$ and identify the annuli at the boundary as in Section 3.5, it is natural to consider the family of metrics $g_{\tau}^{\sharp}$ as being defined on $X$.

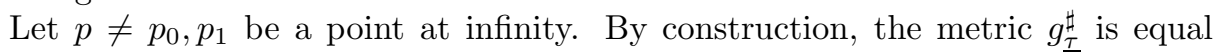
to $g$ on small enough neighborhood of $p$ provided $\underline{\tau}$ is small enough. One can use the fact that the metric $g$ is ACH as in [2, Section I.3] to show that the metric $g$ is $C^{k}$ commensurate with the complex hyperbolic metric on such a neighborhood. In a neighborhood of $p_{0}$ or $p_{1}$, Proposition 3.4 .1 shows that the metric $g_{\tau}^{\sharp}$ is $C^{2}$ commensurate with the complex hyperbolic metric, with uniform constant (i.e. independent of $\underline{\tau}$ provided it is small enough). It follows that the metric $g_{\tau}^{\sharp}$ on $X$ has injectivity radius $i n j_{X}$ bounded below, and that it can be covered by a countable collection of open sets $B_{l}$, which are identified to the complex hyperbolic ball $B(0, \varepsilon)$ of radius $\varepsilon$. In addition, the pullback of $g_{\tau}^{\sharp}$ on $B_{\varepsilon}$ is $C^{k}$-commensurate (with constants independent of $l$ and $\underline{\tau}$ ) with the complex hyperbolic metric. In particular, we have the properties (3.19) and (3.20) on $B_{l}$.

So the only thing left to do is to show that we can use the balls $B_{l}$ to cover $X_{\tau}^{\sharp}$. For that we must discard sufficiently many balls. It is easy to check that the subfamily $B_{l}$ indexed by

$$
\left\{l \quad \mid B_{l} \subset \widetilde{X}_{\underline{\tau}}=X \backslash\left(B_{\lambda_{0}^{0}}\left(p_{0}\right) \cup B_{\lambda_{0}^{1}}\left(p_{1}\right)\right)\right\}
$$

covers $X_{\underline{\tau}}^{\sharp}$ for all $\underline{\tau}$ sufficiently small, and that the restriction of the canonical projection from each ball to $X_{\underline{\tau}}^{\sharp}$ is an embedding. Therefore the injectivity radius of $X_{\tau}^{\sharp}$ is uniformly bounded below and we have the uniform controls (3.19) and (3.20) on each ball.

3.6. Weight functions. It is important to define suitable weight functions on $X^{\sharp}$, because weighted Hölder spaces play an essential role in the deformation theory for ACH Einstein metrics (cf. [2]).

In Section 3.3, we constructed a particular coordinate system near $p_{j} \in \partial \bar{X}$. A neighborhood $B_{c}\left(p_{j}\right)$ of $p_{j}$ is identified via these coordinates to $B_{c}^{+} \subset \mathbb{C H}^{n}$ for some $\varepsilon>0$. On $B_{c}^{+}$, we have a particular function given by $f$. This is a defining function for the boundary near $p_{j}$. We can always extend $f$ into a smooth function on $\bar{X}$ which is a defining function for the boundary $Y$. We denote such an extension $f$ as well.

As we see in the definition of an $\mathrm{ACH}$ metric, the weight function

$$
w=\sqrt{f}
$$

on $X$ plays an important role in the analysis.

Then, we ought to explain what is a suitable (sequence) of weight functions on the surgered manifold $X^{\sharp}$. We begin by defining a special function $\hat{f}$ on $\mathbb{C H}^{n}$ which is a smoothing of the function $\left.f\right|_{B_{1}^{+}}$extended on the other side $B_{1}^{-}$by asking whether it is invariant under the inversion $I_{1}$. In horospherical coordinates $(u, v, W)$, one has

$$
I_{1}^{*} u=\frac{u}{\left(u+\frac{1}{4}|W|^{2}\right)^{2}+v^{2}},
$$

$D_{1}=\partial B_{1}^{+}$has equation $\left(u+\frac{1}{4}|W|^{2}\right)^{2}+v^{2}=1$, and $I_{1}^{-1}\left(D_{\lambda}\right)=D_{\lambda^{-1}}$, so the solution is easy: we define

$$
\hat{f}=u \varpi\left(\left(u+\frac{1}{4}|W|^{2}\right)^{2}+v^{2}\right),
$$


where $\varpi$ is a smooth decreasing function so that $\varpi(x)=1$ for $x \leq 1-\varepsilon$ and $\varpi(x)=\frac{1}{x}$ for $x>1+\varepsilon$. Replacing $\hat{f}$ by $\hat{f}+K_{1}^{*} \hat{f}$, we can arrange $\hat{f}$ so that it is invariant under the inversion. Note that $\hat{f}$ is a smooth defining function for $\mathbb{C} \mathcal{H}^{n}$.

Now pass to the weight function. We start from $w=\sqrt{f}$ that was just defined on $\bar{X}$. Given parameters $\underline{\tau}=\left(\lambda_{i}^{j}, \tau_{j}^{i}\right)$ small enough, verifying the compatibility conditions (3.15), (3.16) and (3.17), we can define the manifold $X_{\tau}^{\sharp}$ as explained in Section 3.5. We start by taking two balls $B_{\lambda_{0}^{j}}\left(p_{j}\right)$, and we identify isometrically the annuli $V_{\left(\lambda_{0}^{j}, \lambda_{1}^{j}\right)}(p)$. We examine more closely how the weight function is transported via this isometry. We can actually identify each annulus to a reference annulus $U_{(1 / K, K)} \subset \mathbb{C} \mathcal{H}^{n}$. In the case of $p_{0}$, we have an isometry

$$
H_{p_{0}}: U_{(1 / K, K)} \rightarrow V_{\left(\lambda_{0}^{0}, \lambda_{1}^{0}\right)}\left(p_{0}\right)
$$

and we see that $H_{p_{0}}^{*}(f)=\left(\lambda_{0}^{0} \lambda_{1}^{0}\right)^{-1 / 2} f$. Similarly, there is an isometry $H_{p_{1}}$ for the other point and $H_{p_{1}}^{*}(f)=\left(\lambda_{0}^{1} \lambda_{1}^{1}\right)^{-1 / 2} f$. Now, we add the condition

$$
\lambda_{0}^{0} \lambda_{1}^{0}=\lambda_{0}^{1} \lambda_{1}^{1}
$$

which, together with the previous compatibility conditions, implies

$$
\lambda_{i}^{0}=\lambda_{i}^{1} \text { for } i=0,1 .
$$

We want to glue together the two functions $H_{p_{i}}^{*} f$ on $U_{(1 / K, K)}$. The solution is to replace both functions by $\left(\lambda_{0}^{0} \lambda_{1}^{0}\right)^{-1 / 2} \hat{f}$, which indeed coincides with $H_{p_{0}}^{*} f$ (resp. $\left.H_{p_{1}}^{*} f\right)$ on $B_{1-\varepsilon}^{+}\left(\right.$resp. $\left.B_{1-\varepsilon}^{-}\right)$. We denote by $f^{\sharp}$ the resulting function on $X^{\sharp}$, and we can define the weight function on $X_{\underline{\underline{I}}}^{\sharp}$ :

$$
w^{\sharp}=\sqrt{f^{\sharp}} .
$$

The usefulness of $w^{\sharp}$ as a weight for all metrics $g_{\underline{工}}^{\sharp}$ comes from the fact that it does not vary too quickly:

Lemma 3.6.1. There is a fixed constant $c$ such that for any $\underline{\tau}$ one has

$$
\sup _{X_{ \pm}^{\sharp}}\left|\nabla \ln w^{\sharp}\right| \leq c .
$$

Proof. This is easy to check in the definition of $w^{\sharp}$.

\section{GLuing}

Starting from an ACH Einstein manifold $(X, g)$, we have constructed a family of approximately ACH Einstein metrics $g_{\tau}^{\sharp}$ on the manifold $X^{\sharp}$ (or $X^{b}$ ) obtained by adding one handle to $X$. We are going to show that, modulo the vanishing of a certain obstruction, one can perturb $g_{\underline{\underline{I}}}^{\sharp}$ in order to get a true Einstein metric.

4.1. Recollection of deformation theory. The deformation theory for asymptotically symmetric metrics can be found in [2. For be a Riemannian metric $h$ on $X$, put

$$
\Phi^{g}(h)=\operatorname{Ric}^{h}+\frac{n+1}{2} h+\left(\delta^{h}\right)^{*}\left(\delta^{g} h+\frac{1}{2} d \operatorname{tr}^{g} h\right) .
$$

It is shown in 2] that, provided $\operatorname{Ric}^{h}<0$ and $\left|\delta^{g} h-\frac{1}{2} d \operatorname{tr}^{g} h\right| \rightarrow 0$,

$$
\Phi^{g}(h)=0 \Leftrightarrow \begin{cases}\operatorname{Ric}^{h}+\frac{n+1}{2} h=0, \\ \delta^{g} h+\frac{1}{2} d \operatorname{tr}^{g} h=0 .\end{cases}
$$


The first equation is of course the Einstein equation, and the other one is interpreted as a gauge condition. Indeed, up to the action of a diffeomorphism, one can always assume that $\delta^{g} h+\frac{1}{2} d \mathrm{tr}^{g} h=0$ for Riemannian metrics close enough to $g$.

The differential of the operator $\Phi^{g}$ at the metric $g$ is given by

$$
\begin{aligned}
d_{g} \Phi^{g} \dot{h}=\frac{1}{2}\left(\nabla^{g}\right)^{*} & \nabla^{g} \dot{h}-\stackrel{\circ}{\mathrm{R}}^{g} \dot{h} \\
& +\frac{1}{2}\left(\operatorname{Ric}^{g} \circ \dot{h}+\dot{h} \circ \operatorname{Ric}^{g}\right)+\frac{n+1}{2} \dot{h},
\end{aligned}
$$

where the action of the curvature $\mathrm{R}$ on symmetric 2 -tensors is given by

$$
(\stackrel{\circ}{\mathrm{R}} \dot{h})_{u, v}=\sum \dot{h}\left(\mathrm{R}_{e_{i}, u} v, e_{i}\right)
$$

for an orthonormal basis $\left(e_{i}\right)$ of $T X$. If $g$ is Einstein, we have the identity

$$
\frac{1}{2}\left(\operatorname{Ric}^{g} \circ \dot{h}+\dot{h} \circ \operatorname{Ric}^{g}\right)+\frac{n+1}{2} \dot{h}=0 .
$$

We are interested in the linearization of the equation $\Phi^{g_{I}^{\sharp}}(h)=0$ at $h=g_{\underline{I}}^{\sharp}$, which gives a formally selfadjoint operator. We will denote it by

$$
L_{\underline{\tau}}=d_{g_{\underline{x}}^{\sharp}} \Phi_{g_{\underline{\tau}}^{\sharp}} .
$$

4.2. Linear theory. The Hölder spaces $C_{\delta}^{k, \alpha}:=\left(w^{\sharp}\right)^{\delta} C^{k, \alpha}$ for functions and more generally for tensors on $X_{\underline{\tau}}^{\sharp}$ are endowed with their usual norms.

From the uniform geometry stated in Lemma 3.5.4 and Lemma 3.6.1, one deduces immediately:

Lemma 4.2.1. There exists a constant $c$, depending on $k$ and $\delta$, such that for any $\underline{\tau}$, one has the uniform local elliptic estimate

$$
\begin{aligned}
\|\dot{h}\|_{C_{\delta}^{k+2, \alpha}} & \leq c\left(\|\dot{h}\|_{C_{\delta}^{0}}+\left\|L_{\underline{\tau}} \dot{h}\right\|_{C_{\delta}^{k, \alpha}}\right), \\
\|\dot{h}\|_{C_{\delta}^{1, \alpha}} & \leq c\left(\|\dot{h}\|_{C_{\delta}^{0}}+\left\|L_{\underline{\tau}} \dot{h}\right\|_{C_{\delta}^{0}}\right) .
\end{aligned}
$$

Of course, one can also define Sobolev spaces. Morally, the $L^{2}$ functions on $X_{\underline{\tau}}^{\sharp}$ are the ones decaying at least as $\left(w^{\sharp}\right)^{n}$. For compatibility of notation, we define

$$
L_{\delta}^{2, k}:=\left(w^{\sharp}\right)^{\delta-n} L^{2, k} .
$$

Notice that with our notation, we have $L^{2, k}=L_{n}^{2, k}$, and $C_{\delta}^{0} \subset L_{\delta^{\prime}}^{2}$ as soon as $\delta^{\prime}<\delta$. We shall need the following lemma for weights on the complex hyperbolic space itself.

Lemma 4.2.2. On $\mathbb{C H} \mathcal{H}^{n}$, the horospherical function $f$ satisfies $f^{\delta / 2} \in L_{\delta^{\prime}}^{2}$ for any $\delta^{\prime}<\delta$ such that $\delta+\delta^{\prime}<n$. The function $\hat{f}$ defined in (3.22) satisfies the same property.

Proof. It is a simple calculation. 
4.3. Linear inverse. The analysis on asymptotically symmetric spaces is developed in [2], and we extract the following theorem.

Theorem 4.3.1 ([2]). The operators

$$
\begin{gathered}
L_{\underline{\tau}}: C_{\delta}^{k+2, \alpha}\left(X_{\underline{\tau}}^{\sharp}\right) \rightarrow C_{\delta}^{k, \alpha}\left(X_{\underline{\tau}}^{\sharp}\right), \\
L_{\underline{\tau}}: L_{\delta}^{2, k+2}\left(X_{\underline{\tau}}^{\sharp}\right) \rightarrow L_{\delta}^{2, k}\left(X_{\underline{\tau}}^{\sharp}\right)
\end{gathered}
$$

are Fredholm for $0<\delta<2 n$. Moreover, their kernel (and cokernel) do not depend on $\delta$, and are identified to the $L^{2}$-kernel (and cokernel) of $L_{\underline{\tau}}$.

Recall that the compatibility conditions (3.15), (3.16), (3.17) and (3.18) for the gluing parameter $\underline{\tau}=\left(\lambda_{k}^{j}, \tau_{k}^{j}\right)$ can be summarized by

$$
\left\{\begin{array}{l}
0<\lambda_{0}^{j}<\lambda_{1}^{j}<\tau_{0}^{j}<\tau_{1}^{j}<1, \\
\tau_{0}^{j}=\tau_{1}^{j} / 2, \text { and } \lambda_{0}^{j}=\left(\lambda_{1}^{j}\right)^{2}, \quad \text { for } \quad j, k=0,1 . \\
\lambda_{k}^{0}=\lambda_{k}^{1}
\end{array}\right.
$$

We will now prove the following key proposition.

Proposition 4.3.2. Assume that the operator $L_{g}$ on $X$ has a trivial $L^{2}$-kernel. Given $\delta \in(0, n)$ and $\alpha \in(0,1)$, there exists a constant $C>0$ such that for all $\underline{\mathcal{I}}$ small enough, verifying the compatibility conditions (4.1), we have

$$
C\|\dot{h}\|_{C_{\delta}^{2, \alpha}} \leq\left\|L_{\underline{\tau}} \dot{h}\right\|_{C_{\delta}^{0, \alpha}} \quad \forall \dot{h} \in C_{\delta}^{2, \alpha}\left(X_{\underline{\tau}}^{\sharp}\right) .
$$

The proposition may be true also for $n \leq \delta<2 n$, but we do not need that since our weight $\delta$ is small. The limitation comes from Lemma 4.2.2.

The proof by a contradiction of this kind in surgery constructions follows a classical scheme. The real case is done in 12 , which we adapt here to the complex case. We give sufficient details, since this is the main technical step for the proof.

Proof. By Lemma 4.2.1, it is sufficient to prove the existence of a uniform constant $C$ such that

$$
C\|\dot{h}\|_{C_{\delta}^{0}} \leq\left\|L_{\underline{\tau}} \dot{h}\right\|_{C_{\delta}^{0}} \quad \forall \dot{h} \in C_{\delta}^{2, \alpha}\left(X_{\underline{\underline{\tau}}}^{\sharp}\right) .
$$

Assume that the proposition is not true. Then, there are sequences $\underline{\tau}_{i}$ verifying (4.1) and $h_{i}$ such that

$$
\underline{\tau}_{i} \rightarrow 0, \quad\left\|h_{i}\right\|_{C_{\delta}^{0}}=1, \quad\left\|L_{\underline{\tau}_{i}} h_{i}\right\|_{C_{\delta}^{0}}=\varepsilon_{i} \rightarrow 0 .
$$

Let $x_{i} \in X_{\underline{\underline{x}}_{i}}^{\sharp}$ be a point at which $\left|\left(w^{\sharp}\right)^{-\delta}\left(x_{i}\right) h_{i}\left(x_{i}\right)\right|=1$. (If $x_{i}$ is on the boundary, then choose an interior point such that $\left|\left(w^{\sharp}\right)^{-\delta}\left(x_{i}\right) h_{i}\left(x_{i}\right)\right| \rightarrow 1$.) Up to extraction of a subsequence, there are basically two cases:

(1) $x_{i}$ converges to an interior point of $X$ or the glued $\mathbb{C} \mathcal{H}^{n}$ (the limit of the $\left.W_{\underline{x}_{i}}\right)$; then we extract a nonzero solution $h$ of $L h=0$ on $X$ or $\mathbb{C} \mathcal{H}^{n}$ and prove that it cannot exist;

(2) $x_{i}$ converges to a boundary point; then there is a sequence of balls around $x_{i}$, with radius going to infinity, which converge to $\mathbb{C} \mathcal{H}^{n}$. Again we extract a nonzero solution of $L h=0$ on $\mathbb{C} \mathcal{H}^{n}$ and prove that it cannot exist.

Let us see that in detail.

In the first case, if the point $x_{i}$ converges to an interior point $x \in X$, then on every compact of $X$ we extract $h_{i} \rightarrow h$, weakly in $C^{1, \alpha}$ and strongly in $C^{0}$. The bounds $\left|h_{i}\right| \leq\left(w^{\sharp}\right)^{\delta}$ and $\left|L_{\mathcal{I}_{i}} h_{i}\right| \leq \varepsilon_{i}\left(w^{\sharp}\right)^{\delta}$ give at the limit on $X$ the conditions

$$
L_{g} h=0, \quad|h(x)|=w(x)^{\delta}, \quad h \in C_{\delta}^{0} .
$$


According to Lemma 4.2.2, the function $w(x)^{\delta}$ is in $L_{\delta^{\prime}}^{2}$ for $\delta^{\prime}>0$ very small. Hence we have $h(x) \in L_{\delta^{\prime}}^{2}$ as well. By assumption the $L^{2}$ kernel of $L_{g}$ is reduced to 0 ; thus we get a contradiction by Theorem 4.3.1.

Still in the first case, if the point $x_{i}$ converges to a point $x$ of the limiting $\mathbb{C} \mathcal{H}^{n}=\lim W_{\underline{\underline{x}}_{i}}$, then on $W_{\underline{\underline{\tau}}_{i}}$ (seen as a standard annulus $U_{\left(1 / K_{i}, K_{i}\right)}$ inside $\mathbb{C H}^{n}$ ) the weight $w^{\sharp}$ coincides with $\mu_{i} \hat{f}^{1 / 2}$ for constants $\mu_{i} \rightarrow \infty$, so that we get the bounds

$$
\left|\mu_{i}^{-\delta} h_{i}\right| \leq \hat{f}^{\delta / 2} \text { and }\left|\mu_{i}^{-\delta} L_{\underline{\tau}_{i}} h_{i}\right| \leq \varepsilon_{i} \hat{f}^{\delta / 2} .
$$

Again, we extract $\mu_{i}^{-\delta} h_{i} \rightarrow h$ on $\mathbb{C H}^{n}$ which is a nonzero solution of $L h=0$ on $\mathbb{C H}^{n}$ with the bound $|h| \leq \hat{f}^{\delta / 2}$. By Lemma 4.2.2, one has $h \in L_{\delta^{\prime}}^{2}$ for $\delta^{\prime}<\delta$, but $L$ on $\mathbb{C} \mathcal{H}^{n}$ has no kernel in $L_{\delta^{\prime}}^{2}$, so we get the contradiction.

In the second case, the idea is to extract (rescaled) $h_{i}$ on larger and larger balls converging to $\mathbb{C} \mathcal{H}^{n}$, but we must see how the weight is transformed. First consider the case where $x_{i}$ goes to a point $p \in \partial X$ which is different from $p_{0}$ and $p_{1}$. As in Section 3.3. we can use horospherical coordinates $(u, v, W)$ near $p$, and the weight $w^{\sharp}$ gets mutually bounded with $\sqrt{u}$. Recall that in this model we have $D_{\alpha}=\partial B_{\alpha}^{+}=\left\{\left(u+\frac{1}{4}|W|^{2}\right)^{2}+v^{2}=\alpha^{2}\right\}$. Define $\alpha_{i} \rightarrow 0$ so that $x_{i} \in D_{\alpha_{i}}$, and, still in horospherical coordinates, pull back all the structures to $B_{\alpha_{i}^{-1}}^{+}$by the parabolic dilation $H_{i}=H_{\sqrt{\alpha_{i}}}$, which sends $B_{\alpha_{i}^{-1}}$ (resp. $\left.B_{1}^{+}\right)$to $B_{1}^{+}$(resp. $B_{\alpha_{i}}^{+}$). Then $H_{i}^{*} u=\alpha_{i} u$ and $y_{i}=H_{i}^{*} x_{i} \in D_{1}$. Therefore the sequence $k_{i}=\alpha_{i}^{-\delta} H_{i}^{*} h_{i}$ on $B_{\alpha_{i}^{-1}}^{+}$ satisfies

$$
\left|k_{i}\right| \leq u^{\delta / 2}, \quad\left|L_{H_{i}^{*} g_{\mathcal{I}_{i}}} k_{i}\right| \leq \varepsilon_{i} u^{\delta / 2}, \quad\left|k_{i}\left(y_{i}\right)\right|=u\left(y_{i}\right)^{\delta / 2},
$$

where $y_{i}=H_{i}^{-1}\left(x_{i}\right) \in D_{1}$ and $H_{i}^{*} g_{\underline{\tau}_{i}}$ goes to the standard metric on $\mathbb{C} \mathcal{H}^{n}$. If $y_{i}$ has a limit in the interior of $D_{1}$, we extract from $\left(k_{i}\right)$ a nonzero limit $k$ such that $L k=0$ and $|k| \leq u^{\delta / 2}$. Therefore $k \in L_{\delta^{\prime}}^{2}$ for $\delta^{\prime}<\delta$, which is a contradiction. If again $y_{i} \in \partial B_{1}^{+}$goes to the boundary of $\mathbb{C H}^{n}$, we reproduce the same process of extraction using dilations from the limit point of $y_{i}$, but the difference is now that the pullbacked points of $y_{i}$ will remain in a compact part of $\mathbb{C} \mathcal{H}^{n}$, and we can conclude in the same way. (One could avoid this double extraction by making a more clever choice of the center of the dilation.)

The last case is when $x_{i}$ tends to $p_{0}$ or $p_{1}$. Let us see that more precisely. We see $x_{i}$ as a point in $\widehat{X}_{\underline{\tau}}=X \backslash \bigcup_{k=0,1} B_{\sqrt{\lambda_{0} \lambda_{1}}}\left(p_{k}\right)$. Here one must be careful that $\lambda_{0}$ and $\lambda_{1}$ also depend on $i$, but we shall omit this dependence. For example, suppose that we are in the case $x_{i} \rightarrow p_{0}$. We identify a small half ball near $p_{0}$ with some $B_{c}^{+}$in $\mathbb{C} \mathcal{H}^{n}$, as in Section 3. If $x_{i}$ is outside the ball $B_{\lambda_{1}}^{+}$, then it is outside the region where the gluing is performed, and we can conclude as above. Suppose on the contrary that $x_{i}$ belongs to the region $B_{\lambda_{1}}^{+}-B_{\sqrt{\lambda_{0} \lambda_{1}}}^{+}$. Then identify this region with an annulus $U_{\left(1 / K_{i}, 1\right)} \subset \mathbb{C} \mathcal{H}^{n}$, with the metric converging to the complex hyperbolic metric. The weight $w_{\underline{I}_{i}}^{\sharp}$ becomes $\mu_{i} \hat{f}^{1 / 2}$ for constants $\mu_{i} \rightarrow \infty$. So one can again conclude as in the beginning of the proof, distinguishing whether $x_{i}$ converges to an interior point or a boundary point of $\mathbb{C} \mathcal{H}^{n}$.

4.4. Gluing Einstein metrics. Here is the technical version of Theorem $\mathrm{A}$ in the Einstein case for $k=1$. The case $k \in \mathbb{N}$ is a trivial generalization making multiple 1-handle surgeries, or by using iteratively Theorem 4.4 .1 together with Proposition 4.5.4. 
Theorem 4.4.1. Fix a weight $\delta<1$. Let $(X, g)$ be an $A C H$ Einstein manifold with $\operatorname{ker}_{L^{2}} L_{g}=0$ and let $g_{\tau}^{\sharp}$ be the sequence of approximate Einstein metrics on $X^{\sharp}$. Then, given $\alpha>0$ small enough, the equation $\Phi^{g_{\perp}^{\sharp}}\left(g_{\underline{工}}^{\sharp}+h\right)=0$ has a unique solution such that $\|h\|_{C_{\delta}^{2, \alpha}} \leq \alpha$ for all $\underline{\tau}$ small enough. In particular $g_{\underline{\tau}}^{\sharp}+h$ is an ACH Einstein metric with conformal infinity $\left(Y^{\sharp}, J_{\underline{\tau}}^{\sharp}\right)$.

A similar statement holds if one replaces $X^{\sharp}$ with $X^{b}$.

Proof. The proof of this result is standard in gluing theory. It is deduced immediately from an effective version of the contraction mapping theorem and Proposition 4.3.2.

Remark 4.4.2. We apparently lost regularity in the theorem, since we started from an ACH Einstein metric with weight $\delta_{0}=1$, and we ended with a slightly smaller weight $\delta<1$. This is an artefact of the proof, and comes from the fact that we used only a rough approximate solution of the Einstein equation near the boundary. Nevertheless, the regularity can be regained a posteriori by applying Proposition 3.2 .2 .

Now pass to the Kähler-Einstein case. We have seen in Section 3.3 .3 that if $X$ is Kähler, then one can make the surgery so that $g_{\tau}^{\sharp}$ remains an ACH Kähler metric on the complex manifold $\left(\bar{X}_{\underline{\tau}}^{\sharp}, J_{\underline{\tau}}\right)$. Moreover, by Proposition 3.4.1, the metrics $g_{\underline{\tau}}^{\sharp}$ are not far from being Kähler-Einstein; in particular they have negative Ricci. It then follows from [6] that there exists on $\left(X_{\underline{\underline{\tau}}}^{\sharp}, J_{\underline{\tau}}\right)$ a complete ACH Kähler-Einstein metric. One deduces immediately

Theorem 4.4.3. If $(X, g)$ is an ACH Kähler-Einstein manifold, then for all $\underline{\tau}$ small enough, $\left(X^{\sharp}, J_{\mathcal{I}}\right)$ admits an ACH Kähler-Einstein metric.

Remark 4.4.4. Instead of using Cheng-Yau's theorem, one can of course directly prove this result: the idea is to keep the complex structure and consider Kähler deformations of the approximate Kähler-Einstein metric compatible with the given complex structure. Hence we are using Proposition 4.3 .2 restricted to Hermitian symmetric 2-tensors. This gluing problem is now automatically unobstructed by Proposition 4.5.2 (cf. below).

We point out that this construction can be carried out in a similar way in the case of $\bar{X}_{\underline{\tau}}^{b}$. The only difference is that the complex structure $J_{\underline{\tau}}$ is now defined only up to sign. However the decomposition in Hermitian and skew-Hermitian tensors still makes sense. Thus, we get the following theorem.

Theorem 4.4.5. If $(X, g)$ is an ACH Kähler-Einstein manifold, then for all $\underline{\tau}$ small enough, $\left(X^{b}, \pm J_{\underline{\tau}}\right)$ admits a locally ACH Kähler-Einstein metric.

Remark 4.4.6. The examples of ACH Einstein manifolds produced by Theorem 4.4.5 are not complex. However they admit a double cover which is ACH Kähler-Einstein. Notice moreover that if the complex dimension $n$ of $X$ is even, then $X^{b}$ is oriented, whereas if $n$ is odd, then $X^{b}$ is nonorientable.

4.5. Obstruction. In this section, we show that the gluing theorem, Theorem 4.4.1, can be used for a large class of ACH Einstein manifolds, and in particular prove Proposition B. The only assumption for the gluing is the vanishing of the obstruction. 
In 2], the following result is proved thanks to a Weitzenböck formula:

Proposition 4.5.1. If $g$ is an $A C H$ (or AH) Einstein metric with negative sectional curvature, then $\operatorname{ker}_{L^{2}} L_{g}=0$.

In particular, this proposition applies to the case of the real and complex hyperbolic space. More generally it shows that any Klein construction (for the real or complex case) gives an unobstructed Einstein metric.

The other vanishing result concerns Kähler-Einstein metrics. All strictly pseudoconvex domains of $\mathbb{C}^{n}$ admit an ACH Kähler-Einstein metric, the Cheng-Yau metric. The following result shows that they are unobstructed for gluing.

Proposition 4.5.2. Let $(g, J)$ be an ACH Kähler-Einstein metric. Then ker $L_{g}$ is identified to infinitesimal complex deformations which leave the CR boundary invariant, in other words, to the compactly supported cohomology group $H_{c}^{1}(X, T X)$.

Proof. The argument is adapted from [1, pp. 362-363], so we will be brief. Decompose a solution $h$ of the equation $L h=0$ into its Hermitian part $h_{H}$ and skew-Hermitian part $h_{S}$. It turns out that the operator $L=\nabla^{*} \nabla-2 \stackrel{\circ}{\mathrm{R}}$ respects this splitting, so that we get

$$
L h_{H}=0 \quad \text { and } \quad L h_{S}=0 .
$$

A Hermitian symmetric 2 -tensor is the same as a $(1,1)$-form, and $L$ is related to the De Rham Laplacian on (1,1)-forms by the formula

$$
L=\Delta-\frac{s}{n} \quad \text { on } \quad \Omega^{1,1}
$$

which obviously has a trivial $L^{2}$-kernel since $s<0$.

On the other hand, a skew-Hermitian symmetric 2-tensor can be identified with a real symmetric endomorphism $\phi$ which anticommutes with $J$. Alternatively, $\phi$ may be considered as a $T^{1,0}$-valued $(0,1)$-form. Now, the operator $L$ is related to the $\bar{\partial}$ operator by the formula

$$
L h=\left(\bar{\partial}^{*} \bar{\partial}+\bar{\partial} \bar{\partial}^{*}\right) \phi
$$

so that an $L^{2}$-solution of $L h=0$ corresponds to a solution of

$$
\bar{\partial} \phi=\bar{\partial}^{*} \phi=0 \text {. }
$$

It follows that $\phi$ represents a symmetric infinitesimal deformation of the complex structure. On the other hand, any infinitesimal complex deformation of the complex structure of a Kähler-Einstein manifold with negative scalar curvature must be symmetric (see [8, Theorem 3.1]), and the proposition is proved.

Our last vanishing result is about dimension 4 . In that case, the metric $g$ may be selfdual. Then one has:

Proposition 4.5.3. If $\left(X^{4}, g\right)$ is an $A C H$ (or AH) self-dual Einstein manifold, then $\operatorname{ker}_{L^{2}} L_{g}=0$.

Proof. We have to prove that there is no $L^{2}$ solution of the equation

$$
L h=\frac{1}{2} \nabla^{*} \nabla h-\stackrel{\circ}{\mathrm{R}} h=0 .
$$

On the trace part, we get

$$
\frac{1}{2} \Delta \operatorname{tr} h-\frac{s}{4} \operatorname{tr} h=0,
$$


which implies $\operatorname{tr} h=0$ since $s<0$. Therefore we are reduced to trace free 2-tensors $h$. In dimension 4 , there is an isomorphism

$$
\Omega_{+}^{2} X \otimes \Omega_{-}^{2} X \stackrel{\sim}{\longrightarrow} S_{0}^{2} T^{*} X,
$$

obtained by sending $\omega^{+} \otimes \omega^{-}$to the 2 -tensor

$$
\left(\omega^{+} \omega^{-}\right)_{u, v}=\left\langle\omega^{+}(u), \omega^{+}(v)\right\rangle .
$$

(Here we identify 2 -forms with skew-Hermitian endomorphisms by sending $u \wedge v$ to the morphism $w \mapsto\langle u, w\rangle v-\langle v, w\rangle u$.) The advantage is to introduce the exterior differential

$$
d_{+}: \Omega^{1} X \otimes \Omega_{-}^{2} X \longrightarrow \Omega_{+}^{2} X \otimes \Omega_{-}^{2} X,
$$

and we shall compare $L$ with the Laplacian $d_{+} d_{+}^{*}$.

Claim. If the metric is Einstein, then on trace free symmetric 2-tensors, one has

$$
\frac{1}{2} \nabla^{*} \nabla-\stackrel{\circ}{\mathrm{R}}=d_{+} d_{+}^{*}-\stackrel{\circ}{\mathrm{W}}--\frac{s}{12} .
$$

The proposition follows immediately from the claim: if the metric is self-dual, then $\mathrm{W}_{-}=0$, and since $s<0$, a solution of $L h=0$ must vanish.

There remains to prove the Claim. One has the Weitzenböck formula on self-dual 2-forms with values in a bundle $E$ with connection [4]:

$$
2 d_{+} d_{+}^{*}=\nabla^{*} \nabla+\frac{s}{3}-2 \mathrm{~W}_{+}+\mathfrak{R}_{+}^{E},
$$

where $\mathrm{W}_{+}$is the Weyl curvature operator acting on 2-forms, and $\mathfrak{R}_{+}^{E}$ denotes some action of the self-dual part of the curvature of $E$. Here, remark that $E=\Omega_{-}^{2}$ is anti-self-dual because the metric is Einstein, so that this term disappears. From the decomposition (still on trace free tensors)

$$
\stackrel{\circ}{\mathrm{R}}=-\frac{s}{12}+\stackrel{\circ}{\mathrm{W}}_{+}+\stackrel{\circ}{\mathrm{W}}_{-},
$$

we deduce

$$
\frac{1}{2} \nabla^{*} \nabla-\stackrel{\circ}{\mathrm{R}}=d_{+} d_{+}^{*}-\frac{s}{12}-\stackrel{\circ}{\mathrm{W}}_{-}+\left(\mathrm{W}_{+}-\stackrel{\circ}{\mathrm{W}}_{+}\right),
$$

so the claim is reduced to proving that $\mathrm{W}_{+}=\stackrel{\circ}{\mathrm{W}}+$ on trace free 2-tensors.

There is only one possible action of self-dual Weyl type tensors on trace free 2-tensors, so there is a constant $\alpha$ such that $\mathrm{W}_{+}=\alpha \stackrel{\circ}{\mathrm{W}}+$ on $S_{0}^{2} T^{*} X$. In order to calculate $\alpha$, it is sufficient to calculate an example. Let us look at a 4-dimensional Kähler manifold, with Kähler form $\omega$, and constant holomorphic sectional curvature (e.g. $\left.\mathbb{C} \mathcal{H}^{2}\right)$. On one hand, one has

$$
\mathrm{W}_{+}(\omega)=\frac{s}{6} \omega .
$$

On the other hand, complete the Kähler form $\omega=\omega_{1}$ into an orthogonal basis $\left(\omega_{1}, \omega_{2}, \omega_{3}\right)$ of $\Omega_{+}^{2}$ with $\left|\omega_{i}\right|=\sqrt{2}$. In this basis, the Weyl tensor is diagonal with eigenvalues

$$
\lambda_{1}=\frac{s}{6}, \quad \lambda_{2}=\lambda_{3}=-\frac{s}{12} .
$$


For any $\xi \in \Omega_{-}^{2}$, we wish to calculate

$$
\begin{aligned}
\stackrel{\circ}{\mathrm{W}}_{+}(\omega \xi)_{u, v} & =\sum_{i=1}^{4}\left\langle\omega \mathrm{W}_{e_{i}, u}^{+} v, \xi e_{i}\right\rangle \\
& =-\frac{1}{2} \sum_{i=1}^{4} \sum_{j=1}^{3} \lambda_{j}\left(\omega_{j}\right)_{e_{i}, u}\left\langle J \omega_{j} v, \xi e_{i}\right\rangle \\
& =\frac{1}{2} \sum_{j=1}^{3} \lambda_{j}\left\langle J \omega_{j} v, \xi \omega_{j} u\right\rangle \\
& =-\frac{1}{2} \sum_{j=1}^{3} \lambda_{j}\left\langle\omega_{j} J \omega_{j} v, \xi u\right\rangle \\
& =\frac{1}{2}\left(\lambda_{1}-\lambda_{2}-\lambda_{3}\right)\langle J v, \xi u\rangle \\
& =\frac{s}{6}(\omega \xi)_{u, v} .
\end{aligned}
$$

Therefore

$$
\stackrel{\circ}{\mathrm{W}}_{+}(\omega \xi)=\frac{s}{6} \omega \xi
$$

and $\alpha=1$, which concludes the proof of the claim.

Finally we check that one can continue the surgeries with the metrics that we construct.

Proposition 4.5.4. The metrics obtained in Theorems 4.4 .1 or 4.4 .3 by surgery from an unobstructed metric are unobstructed for $\underline{\tau}$ small enough.

Proof. In the case of an ACH Kähler-Einstein metric, we obtain it by Theorem 4.4.3. and the resulting metric is automatically unobstructed by Proposition B

Let $g_{\underline{\tau}}^{E}=g_{\underline{\tau}}^{\sharp}+h_{\underline{\tau}}$ be the metric produced by Theorem 4.4.1 and put

$$
L_{\underline{\tau}}^{E}:=d_{g_{\underline{I}}^{E}} \Phi^{g_{\underline{\tau}}^{E}} .
$$

For $\underline{\tau}$ small enough, $\left\|h_{\mathcal{\tau}}\right\|_{C_{\delta}^{2, \alpha}}$ becomes arbitrarily small. Therefore, we can assume that

$$
\left\|\left(L_{\underline{\tau}}-L_{\underline{\tau}}^{E}\right) k\right\|_{C_{\delta}^{0, \alpha}} \leq \frac{C}{2}\|k\|_{C_{\delta}^{2, \alpha}}
$$

for all $k$, where $C$ is the constant of Proposition 4.3.2, Applying Proposition 4.3.2 we deduce

$$
\frac{C}{2}\|k\|_{C_{\delta}^{2, \alpha}} \leq\left\|L_{\tau}^{E} k\right\|_{C_{\delta}^{0, \alpha}}
$$

It follows that the metric $g_{\underline{\tau}}^{E}$ is unobstructed for every $\underline{\tau}$ small enough.

4.6. The $\nu$ invariant. In this section we prove Proposition $\mathrm{D}$ stated in the Introduction, concerning the behavior of the $\nu$ invariant under surgery.

Let $(Y, J)$ be a CR 3-manifold. We pick a pair of points $p_{0}, p_{1} \in Y$. We construct a family of almost complex structures $J_{\underline{\tau}}$ on $Y$ as defined in Section 3.3.2, By definition $J_{\mathcal{\tau}}$ converges smoothly to $J$ as $\tau \rightarrow 0$. By continuity of the $\nu$ invariant we therefore have

$$
\nu\left(J_{\underline{\tau}}\right) \rightarrow \nu(J) \quad \text { as } \quad \underline{\tau} \rightarrow 0 .
$$


The definition of $\nu\left(J_{\underline{\tau}}\right)$ requires the construction of a formal ACH KählerEinstein metric $g_{\underline{\tau}}$ on $\bar{M}:=(0, \eta] \times Y$ with conformal infinity $J_{\underline{\tau}}$ on $\{0\} \times Y$ (cf. [3]). Since $\bar{J}_{\underline{\tau}}$ is given by the standard spherical CR structure in a neighborhood $U_{j} \subset Y$ of $p_{j}$, one can take $g_{\underline{\tau}}$ to be given by the complex hyperbolic metric on $(0, \eta] \times U_{j} \subset M$. One can perform the Klein construction at the points $\left(0, p_{j}\right) \in \bar{M}:=[0, \eta] \times Y$, thus obtaining a manifold $\bar{M}_{\underline{\tau}}^{\sharp}$ with ACH metric $g_{\underline{\tau}}^{\sharp}$ deduced from $g_{\underline{\tau}}$. Topologically $\bar{M}_{\underline{\tau}}^{\sharp}=\bar{M} \cup\{1$-handle $\}$, and the manifold $\bar{M}_{\underline{\tau}}^{\sharp}$ has two boundaries:

- the boundary at infinity $Y^{\sharp}$, obtained by the 1 -handle surgery at $p_{0}, p_{1}$, with conformal infinity $\left(Y^{\sharp}, J_{\underline{\underline{T}}}^{\sharp}\right)$;

- the inner boundary $\{\eta\} \times Y$.

Then, according to [3],

$$
\begin{array}{r}
\frac{1}{8 \pi^{2}} \int_{M_{\underline{\underline{\tau}}}^{\sharp}}\left(3\left|W_{-}\left(g_{\underline{\tau}}^{\sharp}\right)\right|^{2}-\left|W_{+}\left(g_{\underline{\tau}}^{\sharp}\right)\right|^{2}-\frac{1}{2}\left|\operatorname{Ric}_{0}\left(g_{\underline{\tau}}^{\sharp}\right)\right|^{2}+\frac{1}{24} \operatorname{Scal}\left(g_{\underline{\tau}}^{\sharp}\right)^{2}\right) \operatorname{vol}_{\underline{\tau}}^{g_{\tau}^{\sharp}} \\
=\chi\left(\bar{M}_{\underline{\tau}}^{\sharp}\right)-3 \tau\left(\bar{M}_{\underline{\tau}}^{\sharp}\right)+\nu\left(J_{\underline{\tau}}^{\sharp}\right)+\text { inner boundary term. }
\end{array}
$$

On the other hand, we have the same formula for the initial metric $g_{\underline{\tau}}$ :

$$
\begin{aligned}
\frac{1}{8 \pi^{2}} \int_{M}\left(3\left|W_{-}\left(g_{\underline{\tau}}\right)\right|^{2}-\left|W_{+}\left(g_{\underline{\tau}}\right)\right|^{2}-\frac{1}{2}\left|\operatorname{Ric}_{0}\left(g_{\underline{\tau}}\right)\right|^{2}+\frac{1}{24} \operatorname{Scal}\left(\mathrm{g}_{\underline{\tau}}\right)^{2}\right) \mathrm{vol}^{g_{\underline{\tau}}} \\
=\chi(\bar{M})-3 \tau(\bar{M})+\nu\left(J_{\underline{\tau}}\right)+\text { inner boundary term. }
\end{aligned}
$$

Now compare the two formulas: because the surgery takes place on a region where $g_{\underline{\tau}}$ is complex hyperbolic (therefore where the integrand of the LHS vanishes), the two LHS's coincide. The two metrics also coincide near the inner boundary, so that the inner boundary terms coincide. Therefore we deduce that

$$
\chi\left(\bar{M}_{\underline{\underline{\tau}}}^{\sharp}\right)-3 \tau\left(\bar{M}_{\underline{\tau}}^{\sharp}\right)+\nu\left(J_{\underline{\underline{\tau}}}^{\sharp}\right)=\chi(\bar{M})-3 \tau(\bar{M})+\nu\left(J_{\underline{\tau}}\right) .
$$

The manifold $M_{\underline{\underline{\tau}}}^{\sharp}$ is obtained from $M$ by a 1-handle addition; hence the signature does not vary and the Euler number decreases by 1 . Therefore

$$
\nu\left(J_{\underline{\underline{\tau}}}^{\sharp}\right)=\nu\left(J_{\underline{\tau}}\right)+1
$$

and eventually, using (4.7),

$$
\lim _{\underline{\tau} \rightarrow 0} \nu\left(J_{\underline{\underline{T}}}^{\sharp}\right)=\nu(J)+1 .
$$

Remark 4.6.1. In the spherical case, there is of course no need to modify the CR structure $J$ near the point at which the surgery is done. The resulting CR manifolds are spherical, and therefore the $\nu$ invariant is independent of the parameter $\underline{\tau}$. So one gets the equality

$$
\nu\left(J_{\underline{\underline{\tau}}}^{\sharp}\right)=\nu(J)+1 .
$$

\section{ACKnowledgments}

We thank Frank Pacard for several useful conversations. We are grateful to Paul Gauduchon who carefully checked the Weitzenböck formula (4.6). We also thank Yasha Eliashberg, Dmitri Panov and Michael Singer for their interest and many enticing discussions. 


\section{REFERENCES}

[1] A. L. Besse. Einstein manifolds, volume 10 of Ergebnisse der Mathematik und ihrer Grenzgebiete (3) [Results in Mathematics and Related Areas (3)]. Springer-Verlag, Berlin, 1987. MR867684 (88f:53087)

[2] O. Biquard. Métriques d'Einstein asymptotiquement symétriques. Astérisque, (265):vi+109, 2000. MR1760319 (2001k:53079)

[3] O. Biquard and M. Herzlich. A Burns-Epstein invariant for ACHE 4-manifolds. Duke Math. J., 126(1):53-100, 2005. MR2110628 (2006g:32034)

[4] J.-P. Bourguignon and H. B. Lawson, Jr. Stability and isolation phenomena for Yang-Mills fields. Comm. Math. Phys., 79(2):189-230, 1981. MR612248(82g:58026)

[5] D. M. J. Calderbank and M. A. Singer. Einstein metrics and complex singularities. Invent. Math., 156(2):405-443, 2004. MR2052611 (2005h:53064)

[6] S. Y. Cheng and S. T. Yau. On the existence of a complete Kähler metric on noncompact complex manifolds and the regularity of Fefferman's equation. Comm. Pure Appl. Math., 33(4):507-544, 1980. MR575736 (82f:53074)

[7] S. S. Chern and J. K. Moser. Real hypersurfaces in complex manifolds. Acta Math., 133:219271, 1974. MR0425155(54:13112)

[8] X. Dai, X. Wang, and G. Wei. On the stability of Kähler-Einstein metrics. math.DG/0504527.

[9] Y. Eliashberg. Topological characterization of Stein manifolds of dimension $>2$. Internat. J. Math., 1(1):29-46, 1990. MR1044658 (91k:32012)

[10] C. L. Fefferman. Monge-Ampère equations, the Bergman kernel, and geometry of pseudoconvex domains. Ann. of Math. (2), 103(2):395-416, 1976. MR0407320 (53:11097a)

[11] W. M. Goldman. Complex hyperbolic geometry. Oxford Mathematical Monographs. The Clarendon Press, Oxford University Press, New York, 1999. Oxford Science Publications. MR 1695450 (2000g:32029)

[12] R. Mazzeo and F. Pacard. Maskit combinations of Poincaré-Einstein metrics. Adv. Math., 204(2):379-412, 2006. MR2249618 (2007e:53052)

[13] N. Mok and S.-T. Yau. Completeness of the Kähler-Einstein metric on bounded domains and the characterization of domains of holomorphy by curvature conditions. In The mathematical heritage of Henri Poincaré, Part 1 (Bloomington, Ind., 1980), volume 39 of Proc. Sympos. Pure Math., pages 41-59. Amer. Math. Soc., Providence, RI, 1983. MR720056 (85j:53068)

[14] A. Weinstein. Contact surgery and symplectic handlebodies. Hokkaido Math. J., 20(2):241251, 1991. MR:1114405 (92g:53028)

Institut de Recherche Mathématique Avancé, UMR 7501 du CNRS, Strasbourg, France

E-mail address: Olivier.Biquard@math.u-strasbg.fr

Department of Mathematics, Imperial College, London, United Kingdom

E-mail address: rollin@imperial.ac.uk 